\title{
Permian to Cretaceous granites and felsic volcanics from SW Vietnam and S Cambodia: Implications for tectonic development of Indochina
}

\author{
Tod Waight $^{\mathrm{a}, *}$, Michael B.W. Fyhn ${ }^{\mathrm{b}}$, Tonny B. Thomsen ${ }^{\mathrm{b}}$, Tran Van Tri ${ }^{\mathrm{c}}$, Lars H. Nielsen ${ }^{\mathrm{b}}$, \\ Ioannis Abatzis ${ }^{\mathrm{b}}$, Dirk Frei ${ }^{\mathrm{d}}$ \\ ${ }^{a}$ Department of Geosciences and Natural Resource Management (Geology Section), Copenhagen University, Øster Voldgade 10, 1350 Copenhagen K, Denmark \\ ${ }^{\mathrm{b}}$ Geological Survey of Denmark and Greenland, GEUS, Øster Voldgade 10, 1350 Copenhagen K, Denmark \\ ${ }^{\mathrm{c}}$ Geological Survey of Vietnam, Department of Geology and Minerals, No 6 Pham Ngu Lao Street, Hoan Kiem District, Hanoi, Viet Nam \\ ${ }^{\mathrm{d}}$ Department of Earth Sciences, University of the Western Cape, Modderdam Road, Bellville 7530, South Africa
}

\section{A R T I C L E I N F O}

\section{Keyword:}

Granite

Rhyolite

Cambodia

Vietnam

Subduction

Zircon

\begin{abstract}
A B S T R A C T
Zircon ages and geochemistry are presented for igneous rocks from SW Vietnam and S Cambodia. Four main age groupings occur: Cretaceous (107-75 Ma), Early Jurassic (195 Ma), Late Triassic (230-222 Ma), and Permian (294-265 Ma). Cretaceous and Jurassic samples are amphibole-bearing biotite granodiorites to biotite granites and occur mainly east of the Kampot Fold Belt. Pre-Cretaceous samples occur within the Kampot Fold Belt and are dominantly felsic volcanics. The rocks are primarily high-K calc-alkaline, weakly peraluminous rhyolites or granites, with similar arc-like trace element patterns. Cretaceous granites are similar to Dalat Zone Granites and formed during Pacific subduction. Two Cretaceous granites have adakite-like signatures. The youngest Cretaceous granite has an A-type signature and may represent post-collision activity following the cessation of subduction. Jurassic granites are also linked to Pacific subduction. Two Triassic samples could be affiliated to similarly aged rocks in the Chanthaburi Terrane, or linked to the Loei Fold Belt and related to closure to the Sa Kaeo back arc. We cannot exclude that they are related to Pacific Plate subduction. Correlation of Permian volcanics to paleo-Tethys subduction and the Sukhothai-Chanthaburi arc is not clear as the Cambodian volcanics are older than Chanthaburi rocks, and there are stratigraphic contrasts between S Cambodia and Chanthaburi. Furthermore, such a correlation requires extension of the Sa Kaeo suture into S Cambodia and SW Vietnam at a high angle to regional structures. We propose that the Permian volcanics are also related to Paleo-Pacific subduction as shown in regional reconstructions of the region.
\end{abstract}

\section{Introduction}

Southeast Asia represents a complex collage of crustal blocks, volcanic arcs and suture zones progressively accreted during convergent tectonics associated with opening and closure of the Paleo-, Meso-, and Ceno-Tethys oceanic basins and subduction of the Paleo-Pacific in the late Paleozoic to Cenozoic (Metcalfe, 2013a, 2021; Hall, 2012) (Fig. 1). This process continues today with the northwards collision of India into Asia and Australia into Southeast Asia (e.g., Hall, 1996; Morley, 2012). Determining the timing and geochemistry of magmatic events has been key to understanding and unravelling tectonic development in such complex settings. The magmatic history of coastal Vietnam, Malaysia and Thailand are relatively well-studied, however the intervening areas of SW Vietnam and S Cambodia have received little attention in the literature and the affinities of the magmatic rocks in this region are poorly constrained - in part due to limited exposure and deep weathering. In this study, we present U-Pb zircon ages and geochemical data for a comprehensive suite of plutonic and volcanic samples ranging in age from Early Permian to Late Cretaceous from the poorly studied regions of SW Vietnam and S Cambodia with the aim of contributing to the characterization of this region, linking its geological evolution with the surrounding and better-characterized regions, and bridging the gaps in the literature.

\section{Regional geology}

The continental core of SE Asia is termed Sundaland and comprises a number of continental blocks in Vietnam, Cambodia, Laos, eastern

\footnotetext{
* Corresponding author.

E-mail address: todw@ign.ku.dk (T. Waight).
} 


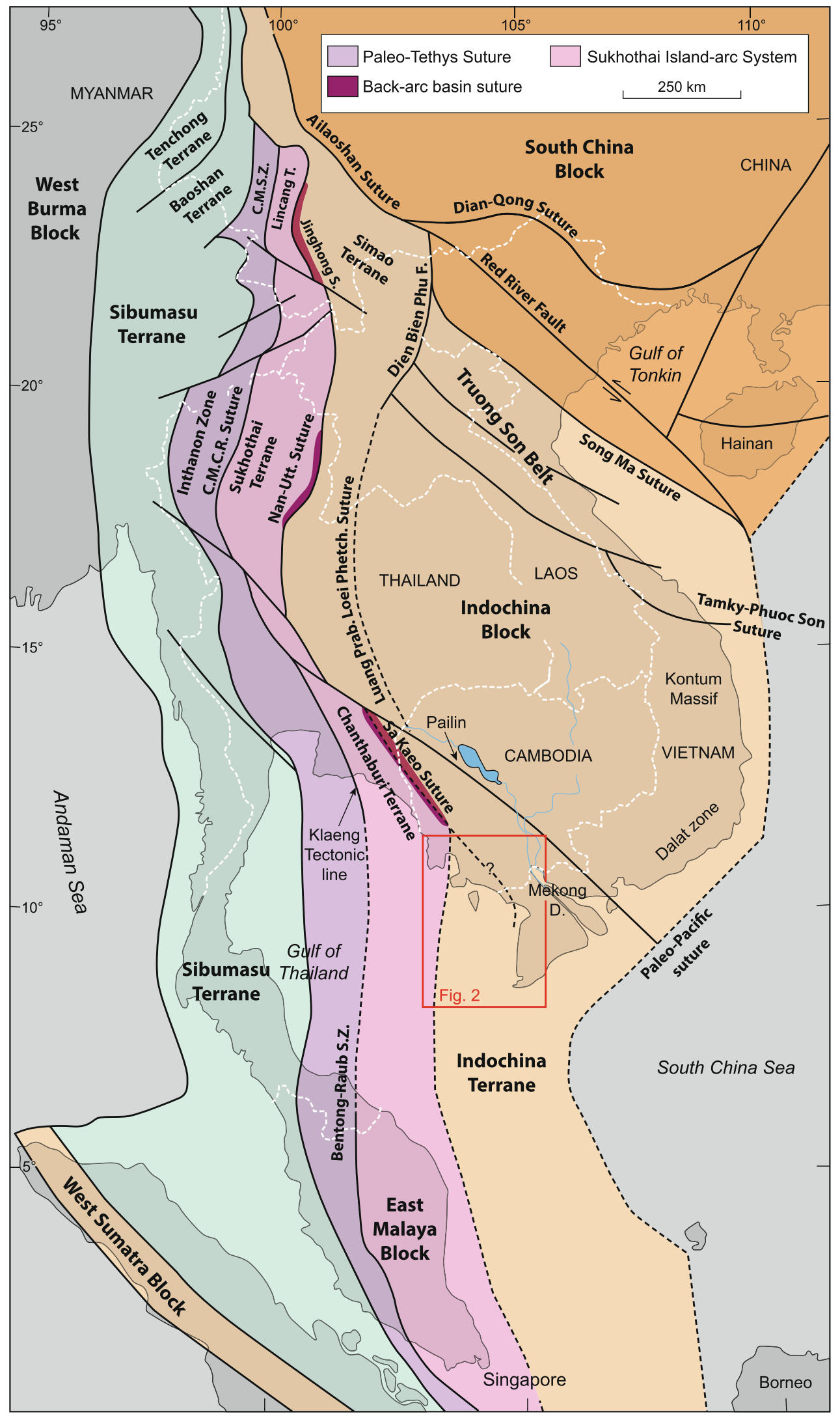

Fig. 1. Regional tectonic map of mainland SE Asia showing the main tectonic units that make up Sundaland. Modified from Metcalfe (2017). Note that extrapolation of the Sa Kaeo Suture, separating the Sukothai Arc from the Indochina Terrane, is uncertain and some models show this extending further to the SE into S Cambodia and S Vietnam (e.g., Wang et al., 2018). Box shows the location of this study (see Fig. 2). 
Thailand, the Sunda Shelf, Sumatra and Borneo, dominated by the Indochina Block in the east (Fig. 1). The basement of the southern Indochina Block comprises granulite facies metamorphic rocks of the Kontum Massif in central Vietnam. These rocks formed in the Proterozoic based on Nd model ages of 1.2-2.4 Ga (Lan et al., 2003), and underwent several metamorphic events, the most recent in the Indosinian Orogeny at ca. $250 \mathrm{Ma}$ (Roger et al., 2000, 2007; Faure et al., 2018; Tran et al., 2020) (Fig. 1).

Within the southeastern part of Vietnam, a Cretaceous magmatic arc forms a younger event dominated by Cretaceous calc-alkaline I-type granitoids of the Dalat Zone, interpreted to have formed above a $\mathrm{W}$ dipping Andean-type subduction zone transitioning into post-orogenic extension (Nguyen et al., 2004; Shellnutt et al., 2013). Evidence for pre-Cretaceous magmatic activity in eastern Indochina is poorly constrained, despite geophysically-based plate tectonic reconstructions suggesting that subduction existed along the eastern margin of Indochina and China since c. Permian times (e.g. Ferrari et al., 2008; Hall, 2012; Metcalfe, 2013a) which is supported by the presence of late Mesozoic I- and A-type granites in SE China (e.g. Sewell and Campbell, 1997; Zhou and Li, 2000; Zhou et al., 2006; Li and Li, 2007).

Western and southern Sundaland (geographically covered by Thailand, Myanmar and Malaysia) is relatively well studied (Sone and Metcalfe, 2008; Searle et al., 2012; Ghani et al., 2013; Zaw et al., 2017). It consists of a series of accreted volcanic arcs and suture zones formed by east to north-eastwards-dipping subduction beneath the Indochina Block and the opening and closing of various permutations of the Tethys Ocean. The easternmost of these terranes is the Sukhothai Arc (also known as the Chanthaburi Terrane) (Sone and Metcalfe 2008, Sone et al. 2012; Metcalfe, 2013b), a volcanic arc formed during northnortheastwards subduction of Paleo-Tethys along the edge of Indochina in the late Carboniferous to Early Permian (Fig. 1). A back arc basin opened in the early-mid-Permian, and then closed again as the Sukhothai Arc was re-accreted in the Triassic (Metcalfe, 2013a; Hara et al., 2018). This event may be linked to Permo-Triassic calc-alkaline magmatism in the Loei Fold Belt (e.g. Nualkhao et al., 2018). It has been suggested that most of the eastern part of the Malay Peninsula is made up of rocks of the Sukhothai Arc (Metcalfe, 2013a, 2017). The boundary between the Sukhothai Arc and Indochina is the remnants of the closed back arc and represented by the Sa Kaeo suture in Thailand (Sra Kaeo in some articles) and a poorly defined cryptic zone east on the Malay Peninsula (Metcalfe, 2013a). To the west of the Sukhothai/Chanthaburi Terrane is the Gondwana-derived Sibumasu Terrane, which was sutured to Indochina following closure of the Paleo-Tethys by the late Permian Early Triassic (Metcalfe, 1996, 2011; Sevastjanova et al., 2011; Qian et al., 2017), an event associated with S-type granitic magmatism and tin mineralization (Searle et al., 2012). The Sibumasu Terrane and the Sukhothai Arc are separated by the Klaeng Tectonic Line in eastern Thailand and the Bentong-Raub Suture on the Malay Peninsula (Sone et al., 2012; Metcalfe, 2013a, 2021) (Fig. 1). The various suture zones represent the remnants of the Paleo-Tethys and are made up of thin zones of mélange, serpentinized ultramafic rocks and oceanic sediments (Metcalfe, 1996, 2013b, 2021).

This study focuses on the region covered by the westernmost (inboard) portions of the late Mesozoic Paleo-Pacific magmatic arc in the east in SW Vietnam, and westwards into the Kampot Fold Belt in southern Cambodia (Fyhn et al., 2010a) (Fig. 2). The Kampot Fold Belt and the late Mesozoic arc are separated by a transitional boundary zone comprising folded pre-Cretaceous strata intruded by various granitic rocks. Further west, the Kampot Fold Belt borders the Late Jurassic to Early Cretaceous Phu Quoc-Kampot Som Basin (Fyhn et al., 2010a). The fold belt consists of folded and thrusted Paleozoic and Mesozoic sediments, volcanics and intrusives dominated by NNW-SSE to N-S trending fold and thrust structures that may have formed during several episodes of deformation, the latest being late Cretaceous to Paleocene thrusting and uplift possibly associated with collisional events around Sundaland (Fyhn et al., 2010a, 2016). The region is tectonically quiescent and has
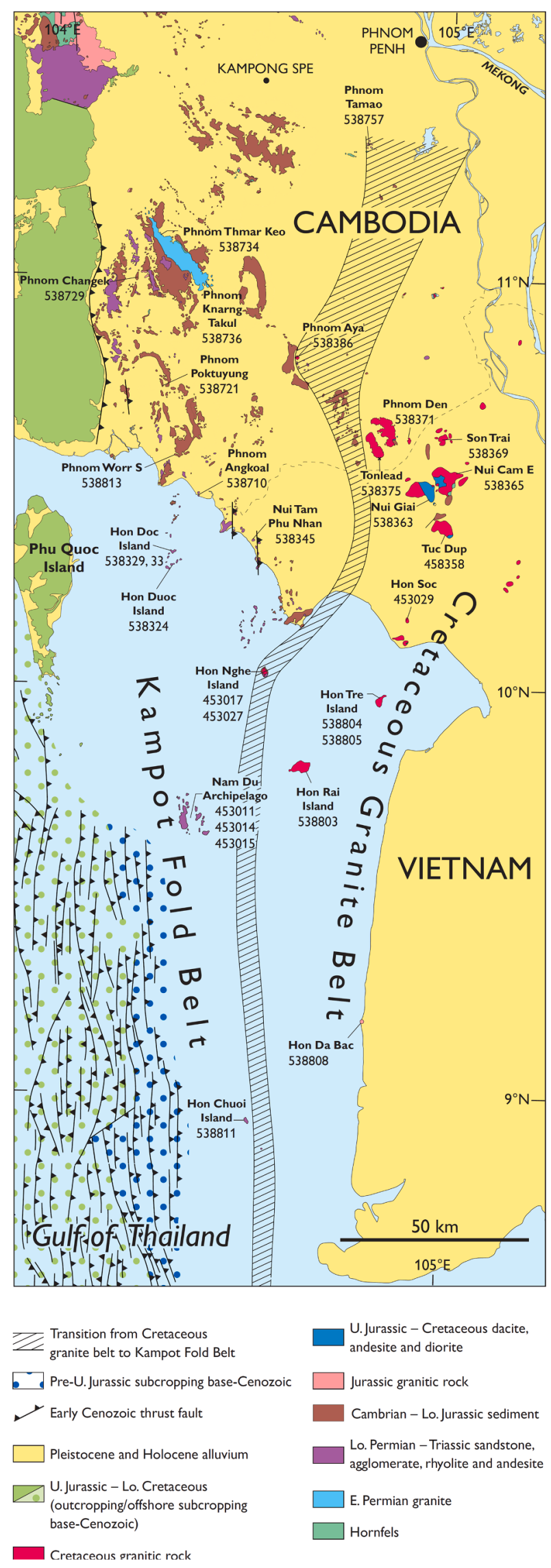

Fig. 2. Sample location map - modified from BRGM (1973) and Fyhn et al. (2016). The striped region represents the transition zone between the late Mesozoic arc to the east and the Kampot Fold Belt to the west. 
been so since the Early Cenozoic, only interrupted by modest pulses of broad uplift in the Late Paleogene and Early Neogene (Fyhn et al., 2016). The landscape consists of scattered, tens to a few hundred meters high, densely vegetated hills and islands that form erosional remnants cored by laterite and weathered Paleozoic-Mesozoic rocks set in a flat-lying Holocene delta plain and shallow sea. Granites are actively quarried in the Vietnamese part of the region allowing sampling of fresh rocks from outcrop. In Cambodia, outcrops of volcanic rocks in general and granites are typically restricted to smaller inactive quarries, road cuts, pits and beach exposures and are highly susceptible to weathering in the tropical moist climate (Fig. 3).

Investigation of the igneous complexes and the pre-Cretaceous deposits in this region is in its infancy. The outline of the Vietnamese part of the study area is summarized by Tran and Vu (2011), and U-Pb zircon ages for volcanic and granitic samples from S Cambodia and Vietnam (including some of the same locations presented here) have been presented by Fyhn et al. (2010a, 2016) and Tran et al. (2020), although no analytical data were presented in these studies. Cheng et al. (2019) present a suite of whole-rock Rb-Sr ages for rocks from NE and $\mathrm{S}$ Cambodia, which spatially overlap in part with the rocks investigated here. Recently, Nong et al. (2021) have provided additional U-Pb zircon ages and geochemical data for granitoids from a portion of the same region as investigated here.

\section{Methods}

Major and trace elements analyses were carried out on rock powders by fusion ICPMS at ACTLABS (www.actlabs.com) using protocols 4B and 4B2. As far as possible, fresh samples were collected during fieldwork from primarily roadcuts and quarries (Fig. 3), however due to ubiquitous and intense tropical weathering and limited outcrop, several samples show clear evidence for weathering and this is evident in their chemical analyses and petrography. Given the scarcity of fresh rocks in widespread areas and the lack of previous data from the region, we choose to include this data, despite the weathering complicating identification of primary signatures.
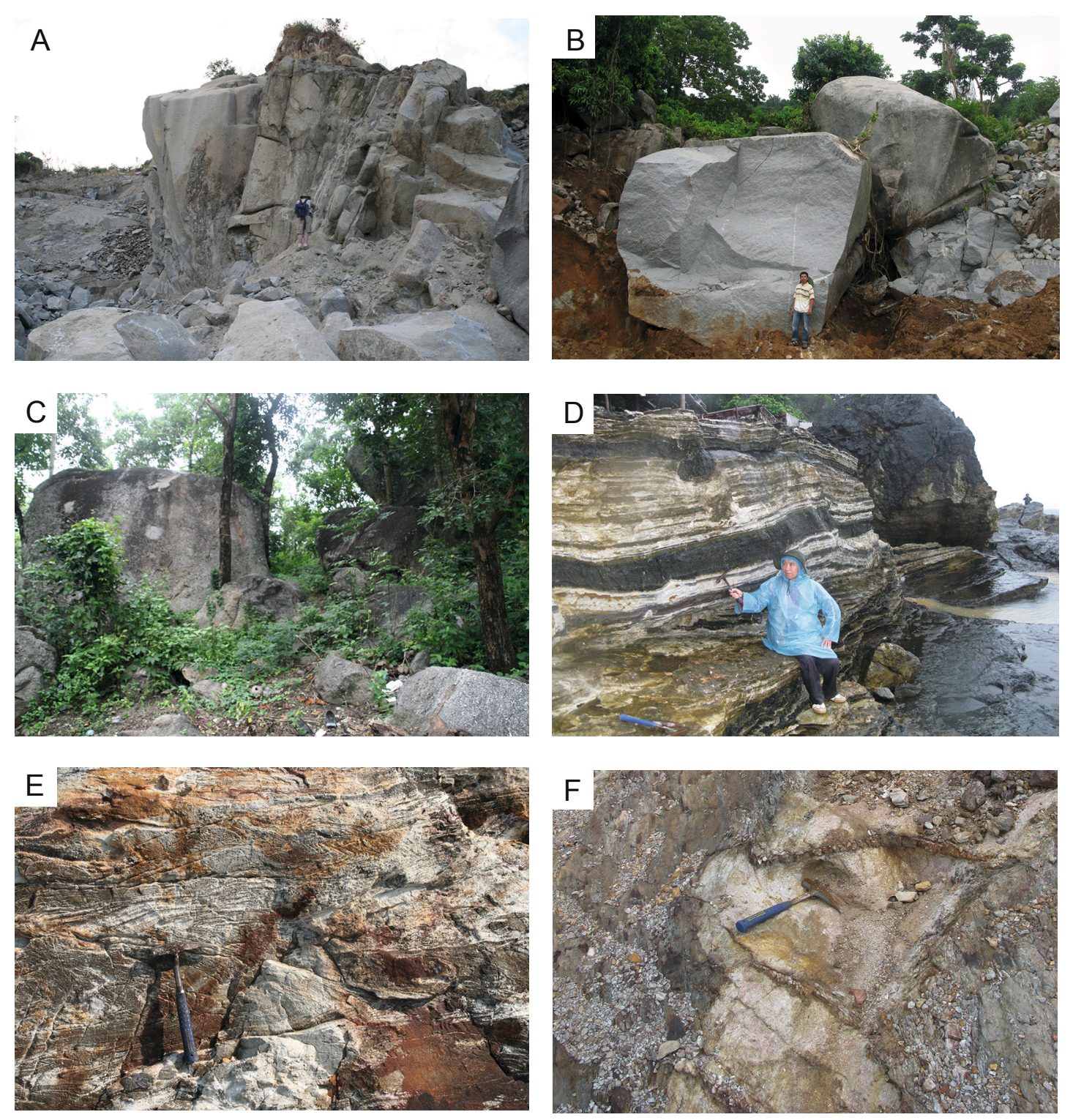

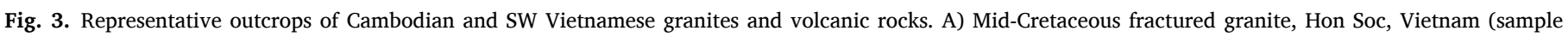
453028); B) Mid-Cretaceous granite weathered out and appearing as a large boulder with a fresh core that is quarried. Hon Tre Island (south), Vietnam (sample 538804); C) Early Jurassic granite, Phnom Tamao, Cambodia (sample 538757); D) Triassic rhyolitic ash flow tuffs deposited subsea and interbedded with lava bombs and chert (Hon Chuoi, sample 538811); E) Slump folded Lower Permian ignimbrite from Nam Du Island, Vietnam (sample 453014); F) Weathered early Permian granite dyke intruding Paleozoic mud- and sandstones. Southern Phnom Worr, Cambodia (sample 538813). 
$\mathrm{U}-\mathrm{Pb}$ zircon analyses on the 538-series samples were carried out by LA-ICPMS at the Geological Survey of Denmark and Greenland (GEUS), Copenhagen using an Element 2 sector field ICPMS coupled to a solidstate $213 \mathrm{~nm}$ laser ablation system. Zircon standard GJ-1 (Jackson et al., 2004) was used for correction of time-dependent instrumental mass bias and for down-hole U-Pb-Th isotopic fractionation. The Plešovice (Slama et al., 2008) and the Harvard 91500 (Wiedenbeck et al., 1995, 2004) zircons were used as secondary quality control standards. Typically, analyses were carried out in sequences including 8-10 unknown zircons, bracketed by three or more standard measurements. Background levels were measured for $30 \mathrm{~s}$ prior to ablation of the zircon for $30 \mathrm{~s}$ using a laser spot size of $25 \mu \mathrm{m}$ and a wash-out period of 20-30 s. Acquired data were processed offline using the Iolite software package $v$. 2.5 (Hellstrom et al., 2008; Paton et al., 2011) and the add-in VizualAge data reduction scheme (Petrus and Kamber, 2012). Zircon analyses showing significant amounts of common lead were corrected using a measured mass 204-procedure including a correction for ${ }^{204} \mathrm{Hg}$ background from the $\mathrm{Ar}+\mathrm{He}$ gas; in general analyses requiring common $\mathrm{Pb}$ correction are not used in most age calculations. Samples from the 453series (previously presented by Fyhn et al. (2010a, 2016) and Tran et al. (2020)) were analyzed using the same instrumentation and similar protocols as described in detail in Gerdes and Zeh (2006) and Frei and Gerdes (2009). All zircon data sets were screened to remove all analyses that had negative rho values, significant common $\mathrm{Pb}$ and/or major discordance, and final dates are determined using only analyses that are within $\pm 5 \%$ of concordance. Ages were calculated using Isoplot version 3.7.1 (Ludwig, 2003). Most samples do not define concordia ages, and are therefore presented as weighted average ${ }^{238} \mathrm{U} /{ }^{206} \mathrm{~Pb}$ ages at $95 \%$ confidence levels. Detailed analytical parameters are summarized in Appendix A.

\section{Results}

\section{1. $U-P b$ zircon geochronology and petrography}

Descriptions of the samples and presentation of the $\mathrm{U}-\mathrm{Pb}$ zircon data (including Tera-Wasserburg plots) are presented in full in Appendix B, with U-Pb data presented in Appendix C, and only a summary is given here. Sample locations are shown in Fig. 2, and representative field locations in Fig. 3. Four main age groupings are identified in this study: Cretaceous (107-75 Ma, 11 samples), Early Jurassic (195 Ma, 2 samples), Late Triassic (230-222 Ma, 2 samples), and Permian (294-265 Ma, 12 samples). New dates and previously published data are summarized in Table 1 . The Cretaceous samples range from amphibolebearing biotite granites and granodiorites ( \pm titanite) to more evolved biotite granites and leucogranites; most are relatively fresh although some show minor alteration such as sericitization of feldspars and chloritization of biotite. The Early Jurassic samples resemble the Cretaceous samples and are biotite granites. The first of the Late Triassic samples is a highly weathered, relatively quartz and muscovite-rich sample of unclear origin, although the presence of a dominantly single-aged zircon population suggests an ultimately igneous origin. The second is a well-bedded subaqueous tuffite. The Permian samples are predominantly fine-grained relatively felsic and leucocratic volcanic samples, some containing feldspar and quartz phenocrysts, and typically displaying evidence for alteration of the fine-grained groundmasses. Also included in the Permian sample group are two variably altered biotite granites, and two granitic dikes all of which intrude Paleozoic sediments.

\subsection{Geochemistry}

Major and trace element analyses of the studied samples are presented in Table 2. All the Triassic and Permian volcanic samples show clear evidence for alteration in hand specimen and in thin section, and this is reflected in a relatively high loss on ignition ( $\mathrm{LOI}=1.5-4.5 \mathrm{wt} \%$ ).
There is a clear correlation between LOI and the Chemical Index of Alteration (CIA of Nesbitt and Young (1982)) (Fig. 4). Nesbitt and Young (1982) define fresh granite as having a CIA of between 45 and 55, and most samples with high LOI in this study have CIA $>55$, indicative of significant weathering and chemical disturbance. Based on these observations, all samples from this study with CIA > 55 are highlighted with a circle in all geochemical plots, and their classification in the totalalkalies - silica diagram and $\mathrm{SiO}_{2}-\mathrm{K}_{2} \mathrm{O}$ diagram is therefore not trustworthy. We note that several samples from the study of Nong et al. (2021) also have CIA > 55 and may be altered, although their LOI is relatively low (Fig. 4).

Most samples are acidic with $\mathrm{SiO}_{2}$ between 67 and $81 \mathrm{wt} \%$, (Cretaceous $=58-76 \mathrm{wt} \%$, Jurassic $=69-72 \mathrm{wt} \%$, Triassic $=67 \mathrm{wt} \%$, Permian $=72-81 \mathrm{wt} \%$ ); one Cretaceous sample is intermediate (538804: Hon Tre S) with $\mathrm{SiO}_{2}=58.3 \mathrm{wt} \%$. Samples with $>77 \mathrm{wt} \% \mathrm{SiO}_{2}$ are altered, as indicated by high LOI and CIA. In the total alkalies-silica diagram (Le Bas et al., 1986; Middlemost, 1994; Fig. 5), most samples are subalkaline and plot in or close to the rhyolite/granite field, with a few samples falling in the dacite/granodiorite field. The Permian and Triassic samples have relatively low $\mathrm{Na}$ and $\mathrm{K}$ reflecting alteration. One Cretaceous sample (53729) plots in the trachydacite (quartz monzonite) field in the TAS diagram and another Cretaceous locality (538804: Hon Tre S) plots in the trachyandesite (monzonite) field. Cretaceous rocks from NE Cambodia (Cheng et al., 2019) are typically more alkaline than similar aged samples from the Dalat Zone and the SW Vietnamese Cretaceous granites of this study. The trend to lower silica contents in the Dalat Zone samples reflects the inclusion of mafic enclaves in the dataset of Shellnutt et al. (2013). With the exception of two Cretaceous samples, all samples are peraluminous and the data set displays a positive correlation between $\mathrm{SiO}_{2}$ and degree of aluminosity (Fig. 6). Several samples with high LOI and CIA $>55$ are very peraluminous with A/CNK between 1.9 and 3.3, reflecting mobility of $\mathrm{Na}$ and $\mathrm{K}$ and $\mathrm{Al}$ during weathering. In a $\mathrm{SiO}_{2}$ vs $\mathrm{K}_{2} \mathrm{O}$ plot (Fig. 7) the Cretaceous, Triassic and Jurassic samples plot within the high-K calc-alkaline field together with published analyses from the Dalat Zone and many from SE Cambodia. The Cretaceous intermediate sample (538804: Hon Tre S) is an exception and has high K contents and plots in the shoshonitic field, together with Cretaceous rocks from NE Cambodia. The Permian samples have lower K contents and mostly plot within the calc-alkaline field. As noted above, the high LOI of these samples indicates that potassium may have been mobile and this categorization is unlikely to reflect the original rock composition prior to weathering.

Our samples mostly scatter around the boundary between the calcalkalic to alkali-calcic fields in the modified alkali-lime index plot of Frost et al. (2001) (Fig. 8), with the single intermediate Cretaceous sample (538804: Hon Tre S) plotting in the alkali field together with some Cretaceous samples from NE Cambodia. Most of the Permian samples plot at lower $\mathrm{Na}_{2} \mathrm{O}+\mathrm{K}_{2} \mathrm{O}-\mathrm{CaO}$, which may reflect alteration, however we note that many apparently unaltered Permian samples from SE Cambodia also plot in the calcic field. Harker diagrams show typical trends of decreasing $\mathrm{Al}_{2} \mathrm{O}_{3}, \mathrm{Fe}_{2} \mathrm{O}_{3}, \mathrm{MgO}$ and $\mathrm{CaO}$ with increasing $\mathrm{SiO}_{2}$ for all age groups. $\mathrm{Na}_{2} \mathrm{O}$ and $\mathrm{K}_{2} \mathrm{O}$ show flat to slightly increasing and generally scattered trends with increasing $\mathrm{SiO}_{2}$. The typically low $\mathrm{CaO}, \mathrm{Na}_{2} \mathrm{O}$ and $\mathrm{K}_{2} \mathrm{O}$ contents, and sometimes high $\mathrm{Al}_{2} \mathrm{O}_{3}$ contents of the altered samples are consistent with disturbance during alteration, with important consequences for classifications based on major elements (see above).

In general, there are large amounts of scatter in trace elements (Fig. 9), however notably $\mathrm{Sr}$ and $\mathrm{Zr}$ show broadly decreasing trends, while $\mathrm{Rb}$ increases with increasing $\mathrm{SiO}_{2}$. The REE and $\mathrm{Y}$ show no clear trends with increasing degrees of evolution. The Permian samples investigated here have generally higher $\mathrm{Ba}$ and lower $\mathrm{Rb}$ than the Cretaceous, Jurassic and Triassic samples (Fig. 9). It is unclear if this is a true magmatic signature, or reflects mobility of the large ion lithophile elements during alteration.

All four age groups show generally similar subduction-related/ average continental crustal trace element signatures in MORB-normalized multi- 
Table 1

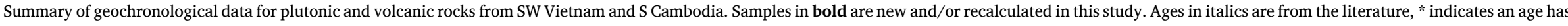
been previously published for this sample, and data are presented and recalculated here.

\begin{tabular}{|c|c|c|c|c|c|c|c|c|c|c|}
\hline Sample & Location & Latitude $^{1}$ & Longitude & Age (Ma) & error $(\mathrm{Ma})^{2}$ & $\begin{array}{l}\text { Intrusive/ } \\
\text { Volcanic }\end{array}$ & Geography & & Method & Source $^{3}$ \\
\hline 538803 & Hon Rai & $09^{\circ} 48^{\prime} 01.5^{\prime \prime}$ & $104^{\circ} 39^{\prime} 10.2^{\prime \prime}$ & 92.3 & 0.7 & I & Mesozoic Granite Belt & Gulf of Thailand & U-Pb zircon & $\mathbf{a}$ \\
\hline 538369 & Son Trai & $10^{\circ} 37^{\prime} 05.2^{\prime \prime}$ & $104^{\circ} 59^{\prime} 39.7^{\prime \prime}$ & 92.3 & 1.3 & I & Mesozoic Granite Belt & SW Vietnam & U-Pb zircon & $\mathbf{a}$ \\
\hline 538809 & Hon Da Bac & $09^{\circ} 10^{\prime} 36.6^{\prime \prime}$ & $104^{\circ} 48^{\prime} 16.9^{\prime \prime}$ & 194.7 & 1.0 & I & Mesozoic Granite Belt & SW Vietnam & U-Pb zircon & $\mathbf{a}$ \\
\hline 538804 & Hon Tre $S$ & $09^{\circ} 57^{\prime} 28.0^{\prime \prime}$ & $104^{\circ} 50^{\prime} 30.1^{\prime \prime}$ & 106.5 & 0.5 & I & Mesozoic Granite Belt & Gulf of Thailand & U-Pb zircon & $\mathbf{a}$ \\
\hline 538375 & Tonleap & $10^{\circ} 33^{\prime} 24.6^{\prime \prime}$ & $104^{\circ} 50^{\prime} 34.0^{\prime \prime}$ & 75.3 & 1.1 & I & Mesozoic Granite Belt & S Cambodia & U-Pb zircon & $\mathbf{a}$ \\
\hline 538371 & Phnom Den & $10^{\circ} 35^{\prime} 59.2^{\prime \prime}$ & $104^{\circ} 54^{\prime} 18.6^{\prime \prime}$ & 96.2 & 0.7 & I & Mesozoic Granite Belt & S Cambodia & U-Pb zircon & $\mathbf{a}$ \\
\hline 538358 & Tuc Dup & $10^{\circ} 22^{\prime} 36.1^{\prime \prime}$ & $104^{\circ} 57^{\prime} 33.3^{\prime \prime}$ & 88.5 & 0.4 & I & Mesozoic Granite Belt & SW Vietnam & U-Pb zircon & $\mathbf{a}$ \\
\hline 538365 & Nui Cam E & $10^{\circ} 30^{\prime} 14.7^{\prime \prime}$ & $105^{\circ} 00^{\prime} 56.2^{\prime \prime}$ & 88.9 & 0.5 & I & Mesozoic Granite Belt & SW Vietnam & U-Pb zircon & $\mathbf{a}$ \\
\hline 538802 & Hon Rai & $09^{\circ} 48^{\prime} 01.5^{\prime \prime}$ & $104^{\circ} 39^{\prime} 10.2^{\prime \prime}$ & 90 & & I & Mesozoic Granite Belt & Gulf of Thailand & U-Pb zircon & $\mathrm{b}$ \\
\hline 538808 & Hon Da Bac & $09^{\circ} 10^{\prime} 36.6^{\prime \prime}$ & $104^{\circ} 48^{\prime} 16.9^{\prime \prime}$ & 195 & & I & Mesozoic Granite Belt & SW Vietnam & U-Pb zircon & $\mathrm{b}$ \\
\hline 538804 & Hon Tre S & $09^{\circ} 57^{\prime} 28.0^{\prime \prime}$ & $104^{\circ} 50^{\prime} 30.1^{\prime \prime}$ & 105 & & I & Mesozoic Granite Belt & Gulf of Thailand & U-Pb zircon & $\mathrm{b}$ \\
\hline 538374 & Tonleap & $10^{\circ} 33^{\prime} 24.6^{\prime \prime}$ & $104^{\circ} 50^{\prime} 34.0^{\prime \prime}$ & 75 & & I & Mesozoic Granite Belt & S Cambodia & U-Pb zircon & $\mathrm{b}$ \\
\hline 538370 & Phnom Den & $10^{\circ} 35^{\prime} 59.2^{\prime \prime}$ & $104^{\circ} 54^{\prime} 18.6^{\prime \prime}$ & 95 & & I & Mesozoic Granite Belt & S Cambodia & U-Pb zircon & $\mathrm{b}$ \\
\hline 538356 & Tuc Dup & $10^{\circ} 22^{\prime} 36.1^{\prime \prime}$ & $104^{\circ} 57^{\prime} 33.3^{\prime \prime}$ & 90 & & I & Mesozoic Granite Belt & SW Vietnam & U-Pb zircon & $\mathrm{b}$ \\
\hline 538368 & Son Trai & $10^{\circ} 37^{\prime} 05.2^{\prime \prime}$ & $104^{\circ} 59^{\prime} 39.7^{\prime \prime}$ & 90 & & I & Mesozoic Granite Belt & SW Vietnam & U-Pb zircon & $\mathrm{b}$ \\
\hline 538364 & Nui Cam E & $10^{\circ} 30^{\prime} 14.7^{\prime \prime}$ & $105^{\circ} 00^{\prime} 56.2^{\prime \prime}$ & 90 & & I & Mesozoic Granite Belt & SW Vietnam & U-Pb zircon & $\mathrm{b}$ \\
\hline \multirow[t]{4}{*}{453028} & Hon Soc & $10^{\circ} 09^{\prime} 05^{\prime \prime}$ & $104^{\circ} 54^{\prime} 12^{\prime \prime}$ & $\mathbf{9 8 . 4}$ & 0.6 & I & Mesozoic Granite Belt & SW Vietnam & U-Pb zircon & $\mathbf{a}^{*}, \mathbf{b}, \mathbf{c}$ \\
\hline & Kirivong & & & 70.56 & 5.58 & I & Mesozoic Granite Belt & S Cambodia & WR Rb-Sr $(\mathrm{n}=2)$ & $\mathrm{e}$ \\
\hline & Ba Phnom & & & 205.19 & 4.88 & I & Mesozoic Granite Belt & S Cambodia & WR Rb-Sr $(\mathrm{n}=5)$ & e \\
\hline & Phnom Da & & & 58.19 & 3.56 & I & Mesozoic Granite Belt & S Cambodia & WR Rb-Sr $(\mathrm{n}=3)$ & e \\
\hline 538386 & Phnom Aya & $10^{\circ} 46^{\prime} 35.9^{\prime \prime}$ & $104^{\circ} 37^{\prime} 48.4^{\prime \prime}$ & 107.3 & 0.4 & I & Transition & S Cambodia & U-Pb zircon & $\mathbf{a}$ \\
\hline 538757 & Phnom Tamao & $11^{\circ} 18^{\prime} 07.4^{\prime \prime}$ & $104^{\circ} 47^{\prime} 59.1^{\prime \prime}$ & 195 & 1.5 & I & Transition & S Cambodia & U-Pb zircon & $\mathbf{a}$ \\
\hline 453027 & Hon Nghe & $10^{\circ} 02^{\prime} 04.0^{\prime \prime}$ & $104^{\circ} 32^{\prime} 49.0^{\prime \prime}$ & 95.2 & 0.9 & I & Transition & Gulf of Thailand & U-Pb zircon & $\mathbf{a}^{*}, \mathbf{b}, \mathbf{c}$ \\
\hline 453017 & Hon Nghe Island & $10^{\circ} 01^{\prime} 20^{\prime \prime}$ & $104^{\circ} 32^{\prime} 50^{\prime \prime}$ & 95.1 & 0.6 & I & Transition & Gulf of Thailand & U-Pb zircon & $\mathbf{a}^{*}, \mathbf{b}, \mathbf{c}$ \\
\hline 538385 & Phnom Aya & $10^{\circ} 46^{\prime} 35.9^{\prime \prime}$ & $104^{\circ} 37^{\prime} 48.4^{\prime \prime}$ & 110 & & I & Transition & S Cambodia & U-Pb zircon & $\mathrm{b}$ \\
\hline \multirow[t]{2}{*}{538756} & Phnom Tamao & $11^{\circ} 18^{\prime} 07.4^{\prime \prime}$ & $104^{\circ} 47^{\prime} 59.1^{\prime \prime}$ & 195 & & I & Transition & Central Cambodia & U-Pb zircon & $\mathrm{b}$ \\
\hline & Phnom Tamoa & & & 182.2 & 43.3 & I & Transition & S Cambodia & WR Rb-Sr $(\mathrm{n}=2)$ & e \\
\hline 538729 & Phnom Changek & $10^{\circ} 58^{\prime} 49.7^{\prime \prime}$ & $104^{\circ} 14^{\prime} 09.9^{\prime \prime}$ & 221.9 & 1.1 & $\mathrm{I}(?)$ & Kampot & S Cambodia & U-Pb zircon & $\mathbf{a}$ \\
\hline 538734 & Phnom Thmar Keo & $11^{\circ} 05^{\prime} 37.2^{\prime \prime}$ & $104^{\circ} 17^{\prime} 10.5^{\prime \prime}$ & 293.4 & 1.9 & I & Kampot & S Cambodia & U-Pb zircon & $\mathbf{a}$ \\
\hline 538721 & Phnom Poktuyung & $10^{\circ} 43^{\prime} 01.3^{\prime \prime}$ & $104^{\circ} 17^{\prime} 54.2^{\prime \prime}$ & 294 & 1.3 & I & Kampot & S Cambodia & U-Pb zircon & $\mathbf{a}$ \\
\hline 538324 & Hon Duoc Island & $10^{\circ} 17^{\prime} 08.5^{\prime \prime}$ & $104^{\circ} 19^{\prime} 19.3^{\prime \prime}$ & 291 & 4.5 & $\mathrm{v}$ & Kampot & SW Vietnam & U-Pb zircon & $\mathbf{a}$ \\
\hline 538333 & Hon Doc Island NE & $10^{\circ} 19^{\prime} 15.9^{\prime \prime}$ & $104^{\circ} 19^{\prime} 57.2^{\prime \prime}$ & 286 & 2.6 & $\mathrm{v}$ & Kampot & SW Vietnam & U-Pb zircon & $\mathbf{a}$ \\
\hline 538329 & Hon Doc Island SE & $10^{\circ} 19^{\prime} 17.4^{\prime \prime}$ & $104^{\circ} 20^{\prime} 13.8^{\prime \prime}$ & 282 & 1.2 & $\mathrm{v}$ & Kampot & SW Vietnam & U-Pb zircon & a \\
\hline 538710 & Phnom Angkoal & $10^{\circ} 27^{\prime} 38.6^{\prime \prime}$ & $104^{\circ} 23^{\prime} 31.8^{\prime \prime}$ & 293.1 & 1.7 & $\mathrm{v}$ & Kampot & S Cambodia & U-Pb zircon & $\mathbf{a}$ \\
\hline 538736 & Phnom Knarng Takul & $11^{\circ} 01^{\prime} 16.2^{\prime \prime}$ & $104^{\circ} 24^{\prime} 12.2^{\prime \prime}$ & 278.1 & 1.4 & I & Kampot & S Cambodia & U-Pb zircon & $\mathbf{a}$ \\
\hline 538728 & Phnom Changek & $10^{\circ} 58^{\prime} 49.7^{\prime \prime}$ & $104^{\circ} 14^{\prime} 09.9^{\prime \prime}$ & 225 & & $\mathrm{~V}$ & Kampot & S Cambodia & U-Pb zircon & $\mathrm{b}$ \\
\hline 538733 & Phnom Thmar Keo & $11^{\circ} 05^{\prime} 37.2^{\prime \prime}$ & $104^{\circ} 17^{\prime} 10.5^{\prime \prime}$ & 295 & & I & Kampot & S Cambodia & U-Pb zircon & $\mathrm{b}$ \\
\hline 538722 & Phnom Poktuyung & $10^{\circ} 43^{\prime} 01.3^{\prime \prime}$ & $104^{\circ} 17^{\prime} 54.2^{\prime \prime}$ & 295 & & I & Kampot & S Cambodia & U-Pb zircon & $\mathrm{b}$ \\
\hline 538323 & Hon Duoc Island & $10^{\circ} 17^{\prime} 08.5^{\prime \prime}$ & $104^{\circ} 19^{\prime} 19.3^{\prime \prime}$ & 290 & & $\mathrm{v}$ & Kampot & Gulf of Thailand & U-Pb zircon & $\mathrm{b}$ \\
\hline 538332 & Hon Doc Island NE & $10^{\circ} 19^{\prime} 15.9^{\prime \prime}$ & $104^{\circ} 19^{\prime} 57.2^{\prime \prime}$ & 285 & & $\mathrm{v}$ & Kampot & Gulf of Thailand & U-Pb zircon & $\mathrm{b}$ \\
\hline 538709 & Phnom Angkoal & $10^{\circ} 27^{\prime} 38.6^{\prime \prime}$ & $104^{\circ} 23^{\prime} 31.8^{\prime \prime}$ & 295 & & $\mathrm{~V}$ & Kampot & S Cambodia & U-Pb zircon & $\mathrm{b}$ \\
\hline 538735 & Phnom Knarng Takul & $11^{\circ} 01^{\prime} 16.2^{\prime \prime}$ & $104^{\circ} 24^{\prime} 12.2^{\prime \prime}$ & 280 & & I & Kampot & S Cambodia & U-Pb zircon & $\mathrm{b}$ \\
\hline 538345 & Nui Tam Phu Nhan & $10^{\circ} 22^{\prime} 57.1^{\prime \prime}$ & $104^{\circ} 27^{\prime} 59.4^{\prime \prime}$ & 287.0 & 1.7 & $\mathrm{v}$ & Kampot & SW Vietnam & U-Pb zircon & $\mathbf{a}^{*}, \mathbf{b}$ \\
\hline 538811 & Hon Chuoi W & $08^{\circ} 57^{\prime} 00.1^{\prime \prime}$ & $104^{\circ} 31^{\prime} 24.2^{\prime \prime}$ & 229.9 & 2.1 & $\mathrm{v}$ & Kampot & Gulf of Thailand & U-Pb zircon & $\mathbf{a}^{*}, \mathbf{b}$ \\
\hline 538813 & Phnom Worr S & $10^{\circ} 33^{\prime} 10.4^{\prime \prime}$ & $104^{\circ} 19^{\prime} 49.8^{\prime \prime}$ & c. 266 & & I & Kampot & S Cambodia & U-Pb zircon & $\mathbf{a}$ \\
\hline 538333 & Hon Doc Island NE & $10^{\circ} 19^{\prime} 15.9^{\prime \prime}$ & $104^{\circ} 19^{\prime} 57.2^{\prime \prime}$ & 284.1 & 1.9 & $\mathrm{~V}$ & Kampot & S Cambodia & U-Pb zircon & $\mathrm{d}$ \\
\hline 538329 & Hon Doc Island SE & $10^{\circ} 19^{\prime} 17.4^{\prime \prime}$ & $104^{\circ} 20^{\prime} 13.8^{\prime \prime}$ & 282.3 & 0.92 & $\mathrm{~V}$ & Kampot & S Cambodia & U-Pb zircon & $\mathrm{d}$ \\
\hline 453014 & Nam Du Island & $09^{\circ} 41^{\prime} 15.0^{\prime \prime}$ & $104^{\circ} 20^{\prime} 59.0^{\prime \prime}$ & 276.9 & 2.0 & $\mathrm{v}$ & Kampot & S Cambodia & U-Pb zircon & $\mathbf{a}^{*}, \mathbf{c}, \mathbf{d}$ \\
\hline
\end{tabular}

(continued on next page) 


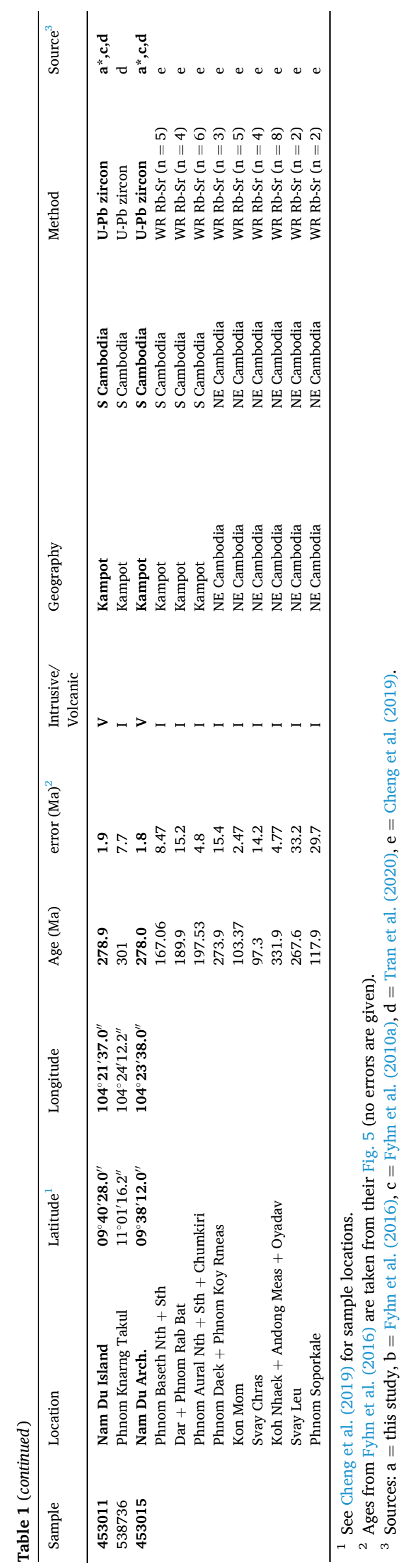

element diagrams with enriched contents of large ion lithophile elements (LILE) and negative troughs at $\mathrm{Nb}$ (Fig. 10). Relative depletions of $\mathrm{Sr}, \mathrm{Ba}$, $\mathrm{P}_{2} \mathrm{O}_{5}$ and $\mathrm{TiO}_{2}$ reflect removal of feldspar, apatite and magnetite during petrogenesis. Two Cretaceous samples are characterized by relatively high $\mathrm{P}_{2} \mathrm{O}_{5}$ (538358: Tuc Dup, 538804: Hon Tre S) - these are the lowest $\mathrm{SiO}_{2}$ samples in the data set. A single Cretaceous sample (538375: Ton Leap) is also distinctive with high contents of Sm, Y and Yb. The Cretaceous, Jurassic and Triassic samples show largely similar trace element patterns to published data from the Dalat Zone granitoids to the northeast, and the recently published data for I-type granites from SW Vietnam and SE Cambodia (Nong et al., 2021). The Permian samples have generally smaller negative $\mathrm{Ba}$ anomalies and slightly lower LILE contents (e.g., $\mathrm{Rb}$ and $\mathrm{Th}$ ) when compared to the Cretaceous samples, although as noted above, given the generally altered nature of the Permian samples and the propensity for mobility of LILE in fluids, it is unclear if this is a primary feature. Compared to published late Paleozoic data from NE Cambodia, the Permian samples investigated here have relatively low $\mathrm{Sr}, \mathrm{P}_{2} \mathrm{O}_{5}, \mathrm{TiO}_{2}$ and HFSE (Nb, Zr, Hf) and higher Ba, reflecting their higher silica contents. We note that we have excluded several samples with unusually low LILE contents, and in some cases low $\mathrm{Zr}+\mathrm{Hf}$ and elevated $\mathrm{TiO}_{2}$ contents from the published Cambodian data (Cheng et al., 2019) plotted on Fig. 10. These samples may be altered and/or be cumulates.

All samples show LREE-enriched REE patterns $\left(\mathrm{La}_{\mathrm{N}} / \mathrm{Lu}_{\mathrm{N}}=3.7-28.4\right)$ and generally relatively flat HREE or depletions in the MREE $\left(\mathrm{Dy}_{\mathrm{N}} / \mathrm{Lu}_{\mathrm{N}}\right.$ $=1.4-0.7$ ) (Fig. 11). Most samples show a negative Eu anomaly (Eu/Eu* $=1.1-0.1$ ) which, together with $\mathrm{La} / \mathrm{Lu}$, decreases with increasing silica content (Fig. 12). The Cretaceous samples mostly exhibit similar REE patterns which resemble analyses of samples from the same region published by Nong et al. (2021), and both data sets tend to have lower HREE than age equivalent samples from the Dalat Zone. Sample 538375 (Tonleap) is clearly distinct with elevated HREE (ca. 50x chondrites) and a pronounced negative Eu anomaly compared to other Cretaceous samples, although we note that similar REE patterns are observed in some Dalat Zone samples. Elevated HREE contents also characterize young post-orogenic S Cambodian samples (e.g., Phnom Da 58 Ma; Cheng et al., 2019) and the A-type granites from SW Vietnam and SW Cambodia of Nong et al. (2021). Samples 538386 and 538804 (Phnom Aya and Hon Tre S, respectively - both c. $107 \mathrm{Ma}$ ) are distinct from the other Cretaceous samples and have similar concave MREE-depleted patterns and relatively small Eu anomalies (Fig. 11). These two samples also have relatively high $\mathrm{Sr} / \mathrm{Y}$ (c. 50) compared to the other Cretaceous samples of this study $(\mathrm{Sr} / \mathrm{Y}<14)$ and this, combined with the negligible Eu anomalies, is a feature of adakitic rocks (e.g., Defant and Drummond, 1990).

The two Jurassic samples have REE patterns that resemble the Cretaceous rocks with relatively flat HREE $\left(\mathrm{Dy}_{\mathrm{N}} / \mathrm{Lu}_{\mathrm{N}}=1.2\right)$, elevated LREE $\left(\mathrm{La}_{N} / \mathrm{Lu}_{\mathrm{N}}=8\right)$ and negative Eu anomalies $\left(\mathrm{Eu} / \mathrm{Eu}^{*}=0.3-0.5\right)$. Their REE patterns are also similar to many rocks of broadly Jurassic age in S Cambodia described by Cheng et al. (2019) (See Fig. 11b), although a few of these show generally enriched REE contents. In contrast, the only Triassic sample analyzed (538729; Phnom Changek) has lower HREE contents $\left(\mathrm{La}_{\mathrm{N}} / \mathrm{Lu}_{\mathrm{N}}=13\right)$, and a smaller negative Eu anomaly (Eu/ $\left.\mathrm{Eu}^{*}=0.8\right)$ (Fig. 11). Although this REE pattern resembles the Cretaceous adakite-like samples described above, the sample has low $\mathrm{Sr} / \mathrm{Y}(2.1)$.

Most Permian samples also exhibit LREE-enriched and flat HREE to depleted MREE patterns with minima at Dy and Ho (Fig. 11). The Permian samples show smaller Eu anomalies at equivalent silica contents compared with the Cretaceous and Jurassic-Triassic samples (Fig. 12); their REE patterns are similar to similarly aged rocks from NE Cambodia (Cheng et al., 2019). Several Permian samples show evidence for disturbance of the REE with both positive and negative Ce anomalies, however these show no clear correlation with indices of alteration such as LOI and CIA.

In the tectonic discrimination diagrams of Pearce et al. (1984), all samples plot in the Volcanic Arc / Syn-collisional Granite (Y vs Nb) and Volcanic Arc Granite ( $\mathrm{Nb}+\mathrm{Y}$ vs $\mathrm{Rb}$ ) fields together with the majority of 
Table 2

eochemical data.

\begin{tabular}{|c|c|c|c|c|c|c|c|c|c|c|c|c|c|c|c|c|c|c|c|}
\hline Sample & $\begin{array}{l}538375 \\
\text { Granite }\end{array}$ & $\begin{array}{l}538358 \\
\text { Granite }\end{array}$ & $\begin{array}{l}538365 \\
\text { Granite }\end{array}$ & $\begin{array}{l}538369 \\
\text { Granite }\end{array}$ & $\begin{array}{l}538803 \\
\text { Granite }\end{array}$ & $\begin{array}{l}538371 \\
\text { Granite }\end{array}$ & $\begin{array}{l}538804 \\
\text { Granite }\end{array}$ & $\begin{array}{l}538386 \\
\text { Rhyolite }\end{array}$ & $\begin{array}{l}538757 \\
\text { Granite }\end{array}$ & $\begin{array}{l}538809 \\
\text { Granite }\end{array}$ & $\begin{array}{l}538729 \\
\text { Granite? }\end{array}$ & $\begin{array}{l}538736 \\
\text { Granite }\end{array}$ & $\begin{array}{l}538329 \\
\text { Rhyolite }\end{array}$ & $\begin{array}{l}538333 \\
\text { Rhyolite }\end{array}$ & $\begin{array}{l}538324 \\
\text { Felsite }\end{array}$ & $\begin{array}{l}538734 \\
\text { Granite }\end{array}$ & $\begin{array}{l}538710 \\
\text { Rhyolite }\end{array}$ & $\begin{array}{l}538721 \\
\text { Granite }\end{array}$ & $\begin{array}{l}538813 \\
\text { Granite }\end{array}$ \\
\hline $\mathrm{SiO}_{2}$ & 74.61 & 67.82 & 73.06 & 76.00 & 72.24 & 77.40 & 58.31 & 70.19 & 71.56 & 69.41 & 66.59 & 73.60 & 76.80 & 73.81 & 72.49 & 78.47 & 73.02 & 80.68 & 76.56 \\
\hline $\mathrm{Al}_{2} \mathrm{O}_{3}$ & 12.71 & 14.40 & 14.15 & 13.20 & 13.53 & 12.54 & 16.28 & 14.31 & 14.35 & 14.20 & 16.00 & 13.42 & 13.70 & 13.71 & 13.58 & 13.26 & 12.35 & 9.65 & 15.62 \\
\hline $\mathrm{Fe}_{2} \mathrm{O}_{3(\mathrm{~T})}$ & 1.55 & 3.79 & 2.06 & 1.16 & 1.93 & 1.39 & 6.66 & 1.77 & 1.80 & 3.42 & 5.79 & 1.77 & 1.54 & 2.06 & 1.94 & 1.27 & 4.60 & 1.48 & 1.11 \\
\hline $\mathrm{MnO}$ & 0.02 & 0.06 & 0.06 & 0.04 & 0.03 & 0.03 & 0.15 & 0.06 & 0.07 & 0.05 & 0.05 & 0.05 & 0.06 & 0.05 & 0.02 & 0.01 & 0.00 & 0.01 & 0.01 \\
\hline $\mathrm{MgO}$ & 0.09 & 1.24 & 0.41 & 0.12 & 0.42 & 0.10 & 1.99 & 0.43 & 0.22 & 0.88 & 0.48 & 0.43 & 0.39 & 0.61 & 0.36 & 0.21 & 0.23 & 0.15 & 0.17 \\
\hline $\mathrm{CaO}$ & 0.61 & 2.68 & 1.41 & 0.62 & 1.34 & 0.20 & 4.95 & 2.64 & 1.10 & 2.39 & 0.08 & 0.18 & 0.11 & 0.09 & 0.16 & 0.04 & 0.03 & 0.21 & 0.04 \\
\hline $\mathrm{Na}_{2} \mathrm{O}$ & 3.22 & 3.34 & 3.79 & 3.51 & 3.14 & 3.53 & 3.39 & 3.29 & 3.64 & 3.04 & 0.37 & 3.82 & 2.28 & 0.74 & 4.53 & 0.07 & 0.13 & 2.87 & 0.14 \\
\hline $\mathrm{K}_{2} \mathrm{O}$ & 4.79 & 4.61 & 4.42 & 4.69 & 4.96 & 4.63 & 5.62 & 3.29 & 4.83 & 3.83 & 3.92 & 3.19 & 3.03 & 3.37 & 2.76 & 3.71 & 3.24 & 2.62 & 3.02 \\
\hline $\mathrm{TiO}_{2}$ & 0.12 & 0.63 & 0.23 & 0.11 & 0.27 & 0.06 & 0.62 & 0.16 & 0.16 & 0.51 & 0.41 & 0.19 & 0.18 & 0.25 & 0.39 & 0.09 & 0.35 & 0.08 & 0.09 \\
\hline $\mathrm{P}_{2} \mathrm{O}_{5}$ & 0.03 & 0.21 & 0.09 & 0.02 & 0.07 & 0.01 & 0.49 & 0.07 & 0.08 & 0.11 & 0.02 & 0.02 & 0.01 & 0.01 & 0.06 & 0.02 & 0.13 & 0.01 & 0.03 \\
\hline LOI & 0.96 & 0.40 & 0.60 & 0.34 & 0.47 & 0.70 & 0.67 & 2.54 & 0.61 & 0.88 & 4.53 & 1.51 & 1.63 & 3.28 & 1.51 & 2.38 & 2.96 & 0.99 & 3.54 \\
\hline
\end{tabular}

Trace elements (ppm)

\begin{tabular}{|c|c|c|c|c|c|c|c|c|c|c|c|c|c|c|c|c|c|c|c|}
\hline Sc & 4 & 8 & 3 & - & 4 & 3 & 15 & 2 & 3 & 10 & 9 & 4 & - & 5 & 8 & 4 & 8 & 7 & 7 \\
\hline $\mathrm{Be}$ & 4 & 4 & 3 & 1 & 2 & 2 & 5 & 2 & 5 & 2 & 2 & 2 & $<1$ & 1 & 1 & 1 & 2 & 1 & 2 \\
\hline $\mathrm{V}$ & 9 & 61 & 17 & 8 & 21 & 6 & 162 & 23 & 9 & 33 & 60 & 16 & 9 & 26 & 24 & 6 & 42 & 5 & 7 \\
\hline $\mathrm{Cr}$ & 20 & 20 & 20 & - & 20 & 30 & 20 & 20 & 20 & 20 & 30 & 20 & - & 20 & 20 & 20 & 20 & 20 & 20 \\
\hline Co & 26 & 17 & 22 & 10 & 25 & 20 & 21 & 5 & 4 & 27 & 7 & 2 & 21.7 & 6 & 30 & 4 & 3 & 6 & 8 \\
\hline $\mathrm{Ni}$ & 20 & 20 & 20 & 1.6 & 20 & 20 & 20 & 20 & 20 & 20 & 20 & 20 & 2.7 & 20 & 20 & 20 & 20 & 20 & 20 \\
\hline $\mathrm{Cu}$ & 10 & 10 & 10 & 3.2 & 10 & 10 & 60 & 10 & 10 & 10 & 20 & 10 & 3.6 & 10 & 10 & 10 & 10 & 10 & 10 \\
\hline $\mathrm{Zn}$ & 30 & 50 & 40 & 18 & 30 & 71.6 & 80 & 30 & 40 & 50 & 70 & 30 & 31 & 30 & 40 & 30 & 0.01 & 40 & 0.01 \\
\hline $\mathrm{Ga}$ & 23 & 19 & 17 & 14.3 & 17 & 17 & 20 & 14 & 22 & 18 & 18 & 13 & 10 & 15 & 12 & 14 & 14 & 13 & 19 \\
\hline $\mathrm{Ge}$ & 2.1 & 1.6 & 1.4 & - & 1.6 & 1.8 & 1.7 & 1.6 & 1.8 & 1.7 & 2 & 1.5 & - & 1.1 & 1.2 & 1.9 & 1.7 & 1.3 & 2.5 \\
\hline As & 11 & 5 & 5 & 0.6 & 5 & 24 & 5 & 5 & 5 & 5 & 73 & 5 & 52 & 5 & 5 & 236 & 79 & 5 & 5 \\
\hline $\mathrm{Rb}$ & 355 & 237 & 167 & 251.9 & 181 & 181 & 171 & 148 & 310 & 149 & 130 & 103 & 74.9 & 116 & 77 & 137 & 154 & 83 & 143 \\
\hline $\mathrm{Sr}$ & 51 & 283 & 193 & 30.8 & 171 & 52 & 972 & 435 & 68 & 116 & 27 & 90 & 93.7 & 194 & 95 & 8 & 77 & 74 & 24 \\
\hline Y & 78.3 & 20.2 & 13 & 11.2 & 16.2 & 17.5 & 21.6 & 7.8 & 28.6 & 22.9 & 13.1 & 13.8 & 10.8 & 20.7 & 27.1 & 22 & 13.5 & 26.8 & 37.6 \\
\hline $\mathrm{Zr}$ & 157 & 271 & 155 & 77.4 & 175 & 84 & 162 & 71 & 112 & 199 & 105 & 99 & 92 & 101 & 149 & 56 & 128 & 109 & 39 \\
\hline $\mathrm{Nb}$ & 14.8 & 14.5 & 11.7 & 13.9 & 9.7 & 13 & 9.8 & 5.1 & 16.5 & 6.6 & 3.8 & 7.8 & 7.1 & 7.8 & 6 & 11.4 & 6.1 & 9.2 & 8.4 \\
\hline Mo & 2 & 2 & 2 & 0.3 & 2 & 2 & 3 & 2 & 2 & 2 & 2 & 2 & 0.5 & 2 & 2 & 2 & 4 & 2 & 2 \\
\hline $\mathrm{Ag}$ & 0.8 & 1.6 & 0.9 & $<0.1$ & 1 & 0.5 & 0.8 & 0.5 & 0.5 & 1.3 & 0.5 & 0.5 & $<0.1$ & 0.6 & 0.6 & 0.8 & 0.5 & 0.5 & 0.5 \\
\hline In & 0.1 & 0.1 & 0.1 & - & 0.1 & 0.1 & 0.1 & 0.1 & 0.1 & 0.1 & 0.1 & 0.1 & - & 0.1 & 0.1 & 0.1 & 0.1 & 0.1 & 0.1 \\
\hline Sn & 5 & 4 & 15 & 3 & 2 & 4 & 2 & 1 & 9 & 4 & 2 & 1 & $<1$ & 1 & 1 & 2 & 1 & 2 & 17 \\
\hline $\mathrm{Sb}$ & 0.2 & 0.4 & 0.2 & $<0.1$ & 0.2 & 0.4 & 0.2 & 0.7 & 0.2 & 0.2 & 7.5 & 0.5 & 0.4 & 0.2 & 0.2 & 9 & 4.9 & 0.2 & 0.2 \\
\hline Cs & 7.2 & 19.8 & 4.8 & 9 & 7.9 & 2.7 & 6.6 & 4.9 & 15.1 & 15 & 4.5 & 3.5 & 2.6 & 4.7 & 1.4 & 5.3 & 3.7 & 1.3 & 3.6 \\
\hline Ва & 199 & 447 & 432 & 49 & 453 & 441 & 821 & 416 & 198 & 372 & 292 & 636 & 509 & 964 & 686 & 233 & 514 & 616 & 201 \\
\hline $\mathrm{La}$ & 42.6 & 49 & 36.4 & 8.8 & 68.3 & 21.1 & 40.3 & 14.1 & 30.6 & 28.7 & 25.9 & 25.8 & 29.7 & 51 & 27.9 & 18.8 & 35.5 & 16.9 & 38 \\
\hline $\mathrm{Ce}$ & 95.5 & 97.1 & 67.9 & 22.1 & 122 & 41.6 & 85.1 & 25.6 & 58.1 & 59.5 & 46.5 & 62.1 & 64.6 & 95.4 & 55.1 & 33.8 & 65.6 & 18 & 65.4 \\
\hline $\operatorname{Pr}$ & 11.8 & 11.2 & 7.41 & 2.52 & 12.6 & 4.73 & 10.6 & 2.87 & 6.44 & 6.71 & 5.71 & 4.79 & 5.28 & 10.5 & 7.34 & 3.63 & 7.61 & 4.35 & 8.25 \\
\hline $\mathrm{Nd}$ & 45.1 & 39.7 & 24.6 & 9.1 & 40.6 & 17 & 43.3 & 10.1 & 21.3 & 26.2 & 20.9 & 15.3 & 17.7 & 34.2 & 27.7 & 12.2 & 28.7 & 16 & 32 \\
\hline $\mathrm{Sm}$ & 11.9 & 7.2 & 4.41 & 2.17 & 6.33 & 3.32 & 9.07 & 1.92 & 4.83 & 5.56 & 3.95 & 2.62 & 2.81 & 5.5 & 5.87 & 2.35 & 5.23 & 3.63 & 6.57 \\
\hline $\mathrm{Eu}$ & 0.363 & 1.34 & 0.707 & 0.24 & 0.84 & 0.294 & 2.3 & 0.583 & 0.546 & 0.815 & 0.963 & 0.522 & 0.51 & 1.11 & 1.52 & 0.31 & 1.29 & 0.595 & 1.19 \\
\hline $\mathrm{Gd}$ & 11.4 & 5.75 & 3.45 & 1.8 & 4.53 & 2.96 & 7.26 & 1.46 & 4.8 & 5.16 & 3.2 & 2.34 & 2.22 & 4.06 & 5.65 & 2.65 & 3.21 & 3.69 & 5.64 \\
\hline $\mathrm{Tb}$ & 2.19 & 0.79 & 0.47 & 0.32 & 0.63 & 0.51 & 0.91 & 0.22 & 0.91 & 0.85 & 0.47 & 0.38 & 0.32 & 0.62 & 0.88 & 0.5 & 0.42 & 0.79 & 1.06 \\
\hline Dy & 13.3 & 4.12 & 2.52 & 2.03 & 3.17 & 2.91 & 4.49 & 1.29 & 5.21 & 4.55 & 2.54 & 2.28 & 1.86 & 3.46 & 4.86 & 3.33 & 2.26 & 5.19 & 6.29 \\
\hline Ho & 2.58 & 0.75 & 0.46 & 0.38 & 0.56 & 0.57 & 0.81 & 0.26 & 1 & 0.92 & 0.45 & 0.46 & 0.42 & 0.63 & 0.95 & 0.71 & 0.44 & 1.05 & 1.26 \\
\hline $\mathrm{Er}$ & 7.96 & 2.2 & 1.37 & 1.16 & 1.79 & 1.56 & 2.4 & 0.81 & 2.81 & 2.5 & 1.35 & 1.45 & 1.27 & 1.92 & 2.81 & 2.23 & 1.38 & 3.36 & 3.56 \\
\hline $\mathrm{Tm}$ & 1.25 & 0.336 & 0.206 & 0.2 & 0.271 & 0.249 & 0.354 & 0.139 & 0.444 & 0.377 & 0.203 & 0.238 & 0.21 & 0.317 & 0.424 & 0.401 & 0.223 & 0.493 & 0.585 \\
\hline $\mathrm{Yb}$ & 7.98 & 2 & 1.32 & 1.39 & 1.73 & 1.75 & 2.16 & 0.98 & 2.97 & 2.42 & 1.26 & 1.69 & 1.48 & 2.26 & 2.83 & 2.66 & 1.48 & 3.1 & 3.94 \\
\hline $\mathrm{Lu}$ & 1.25 & 0.297 & 0.21 & 0.21 & 0.258 & 0.26 & 0.321 & 0.173 & 0.442 & 0.369 & 0.213 & 0.282 & 0.26 & 0.376 & 0.448 & 0.395 & 0.244 & 0.463 & 0.576 \\
\hline $\mathrm{Hf}$ & 5.1 & 6.4 & 3.9 & 2.8 & 4.5 & 2.8 & 4.2 & 1.8 & 3.5 & 5 & 2.6 & 2.5 & 2.7 & 3 & 3.5 & 2.1 & 2.8 & 3.5 & 1.3 \\
\hline Тa & 2.46 & 1.52 & 1.23 & 1.7 & 1.11 & 1.32 & 0.83 & 0.68 & 2.79 & 0.67 & 0.5 & 0.86 & 0.6 & 0.87 & 0.6 & 1.21 & 0.58 & 0.77 & 1.14 \\
\hline W & 135 & 59.9 & 108 & 47.1 & 122 & 96.7 & 46.3 & 17.5 & 12.2 & 111 & 15.3 & 2.5 & 84.2 & 15.3 & 143 & 19.9 & 14.7 & 28.9 & 42.7 \\
\hline $\mathrm{Tl}$ & 1.54 & 1.11 & 0.81 & 0.1 & 1.05 & 0.9 & 0.69 & 0.85 & 1.27 & 0.87 & 0.53 & 0.6 & $<0.1$ & 0.4 & 0.44 & 1.33 & 1.36 & 0.56 & 0.68 \\
\hline $\mathrm{Pb}$ & 23 & 18 & 23 & 11.7 & 22 & 31 & 17 & 7 & 30 & 22 & 48 & 16 & 29.9 & 13 & 5 & 6 & 18 & 11 & 32 \\
\hline $\mathrm{Bi}$ & 0.1 & 0.3 & 0.1 & 0.7 & 0.1 & 6.8 & 0.2 & 0.2 & 1.1 & 0.1 & 0.8 & 0.1 & $<0.1$ & 0.2 & 0.1 & 0.2 & 0.1 & 0.4 & 6 \\
\hline Th & 35.9 & 30.2 & 14.6 & 33.7 & 34.9 & 12.2 & 16.4 & 8.67 & 27.2 & 15.6 & 7.89 & 10.6 & 8.3 & 10.2 & 7.34 & 9.15 & 7.21 & 10.6 & 5.59 \\
\hline $\mathrm{U}$ & 9.58 & 4.35 & 3.28 & 7.7 & 13.2 & 2.65 & 5.89 & 4.69 & 8.86 & 2.9 & 1.99 & 1.97 & 2.3 & 2.86 & 2.48 & 2.34 & 2.21 & 2.56 & 2.93 \\
\hline
\end{tabular}




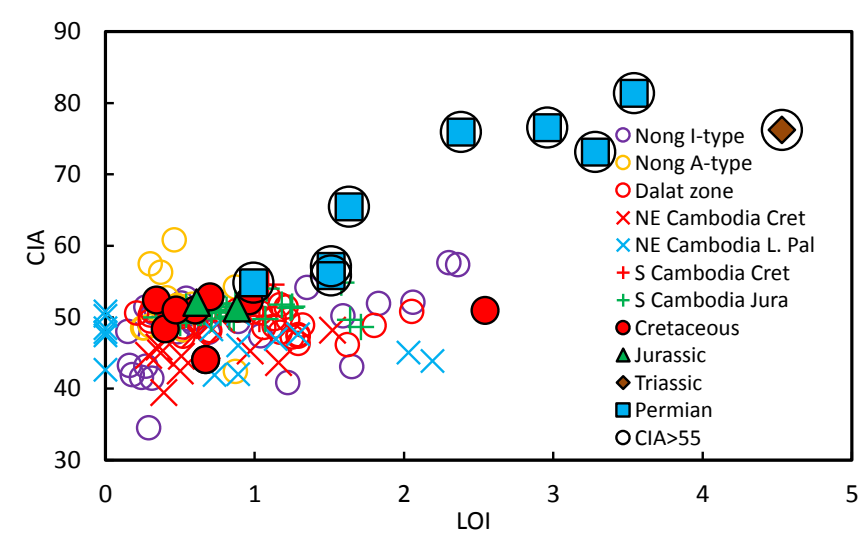

Fig. 4. Plot of Chemical Index of Alteration (CIA of Nesbitt and Young 1982) versus Loss on Ignition (LOI) indicating geochemical evidence for alteration in some samples. Nesbitt and Young (1982) suggest fresh basalt and granite should have a CIA between $30-45$ and 45-55, respectively. Solid symbols represent new data presented in this study. Data from the Dalat Zone are from Thuy et al. (2004) and Shellnutt et al. (2013), and for Cretaceous I- and A-type granites from SW Vietnam and SE Cambodia are from Nong et al. (2021). Data from NE and S Cambodia are from Cheng et al. (2019) and are loosely subdivided based on age into NE Cambodia Cretaceous (c. 117-97 Ma: Svay Chras, Kon Mom, Phnom Soporkaley), NE Cambodia Late Paleozoic (c. 332-274 Ma: Phnom Deak, Ph. Koy Rmeas, Koh Nheak, Andong Meas, Oyadav, Svay Leu), S Cambodia Cretaceous (58-71 Ma: Kirivong, Phnom Da) and S Cambodia Jurassic (167-200 Ma: Ph. Bassett North and South, Dar, Ph. Aural North and South, Ph. Tamao, Ba Phnom, Ph Rab Bat, Ratanakmundol, Chum Kiri). Note that some of these analyses are presented without LOI values.

samples from the Dalat Zone and NE Cambodia (Fig. 13). A clear exception is youngest Cretaceous sample 538735 (Tonleap, $75 \mathrm{Ma}$ ) which plots in the Within-Plate Granite field, reflecting its elevated HREE and Y contents. Several other Cretaceous-Jurassic samples from the literature also plot in the Within Plate Granite field.

\section{Discussion}

\subsection{Geographic distribution}

Four distinct age groupings are identified in this study: Cretaceous (107-75 Ma, $\mathrm{n}=12$ ); Jurassic (195 Ma, $\mathrm{n}=2$ ); Triassic (230-222 Ma, $\mathrm{n}$ =2); and Permian (294-265 Ma, $\mathrm{n}=12$ ). These age groupings show a clear geographic distribution with all Cretaceous rocks occurring in the east, i.e. within the Cretaceous granite belt or along its western margin in the transition zone to the Kampot Fold Belt as defined by Fyhn et al. (2010a). Only two Jurassic samples are identified in this study, one of which occurs at the south of the Cretaceous Granite Belt (538809, Hon Da Bac, 194.7 Ma) and the other in the northern portion of the transition zone to the Kampot Fold Belt (538757, Phnom Tamao, $195 \mathrm{Ma}$ ). However, Cheng et al. (2019) identify several Jurassic (c. 200-170 Ma) intrusives further to the west in the Kampot Fold Belt, suggesting lithologies with this age are more widespread in the province. The

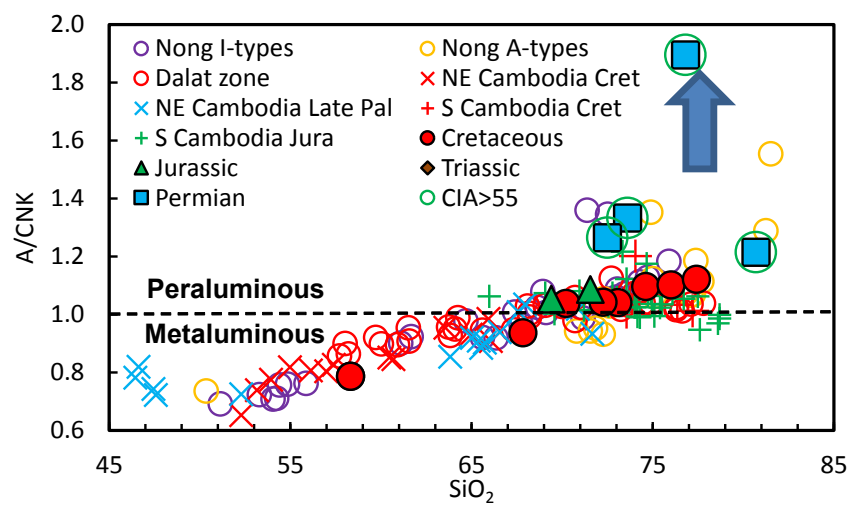

Fig. 6. $\mathrm{SiO}_{2}$ vs aluminium saturation index $(\mathrm{A} / \mathrm{CNK}=\operatorname{molar} \mathrm{Al} /(\mathrm{Ca}+\mathrm{Na}+$ $\mathrm{K})$ ). Note that several altered samples have high values of A/CNK (up to 3.3) and plot off the diagram.

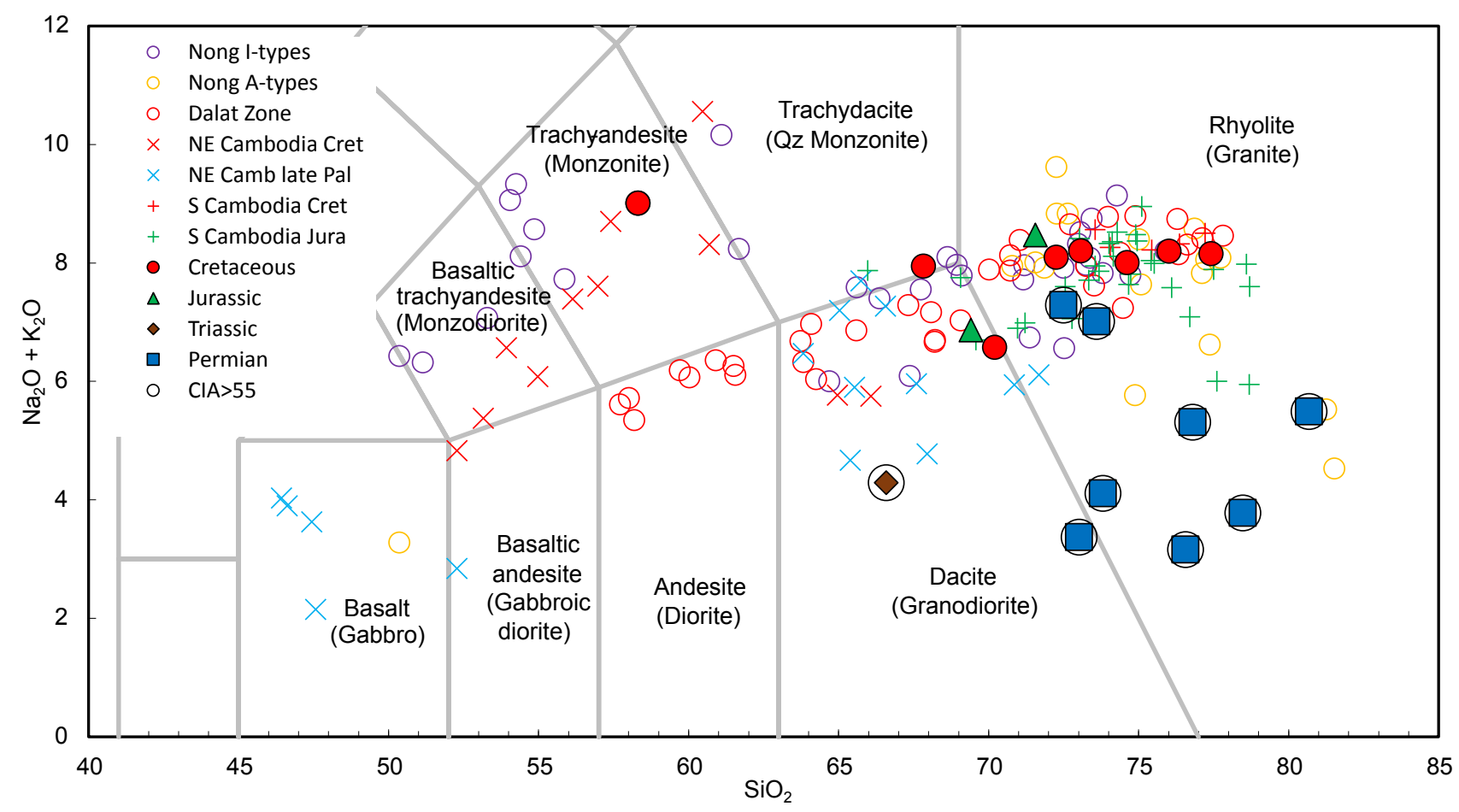

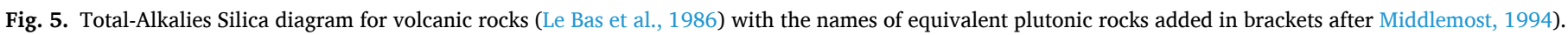
N.B. analyses are plotted as measured (i.e. not corrected to $100 \%$ anhydrous), note the low $\mathrm{Na}+\mathrm{K}$ in altered samples. 

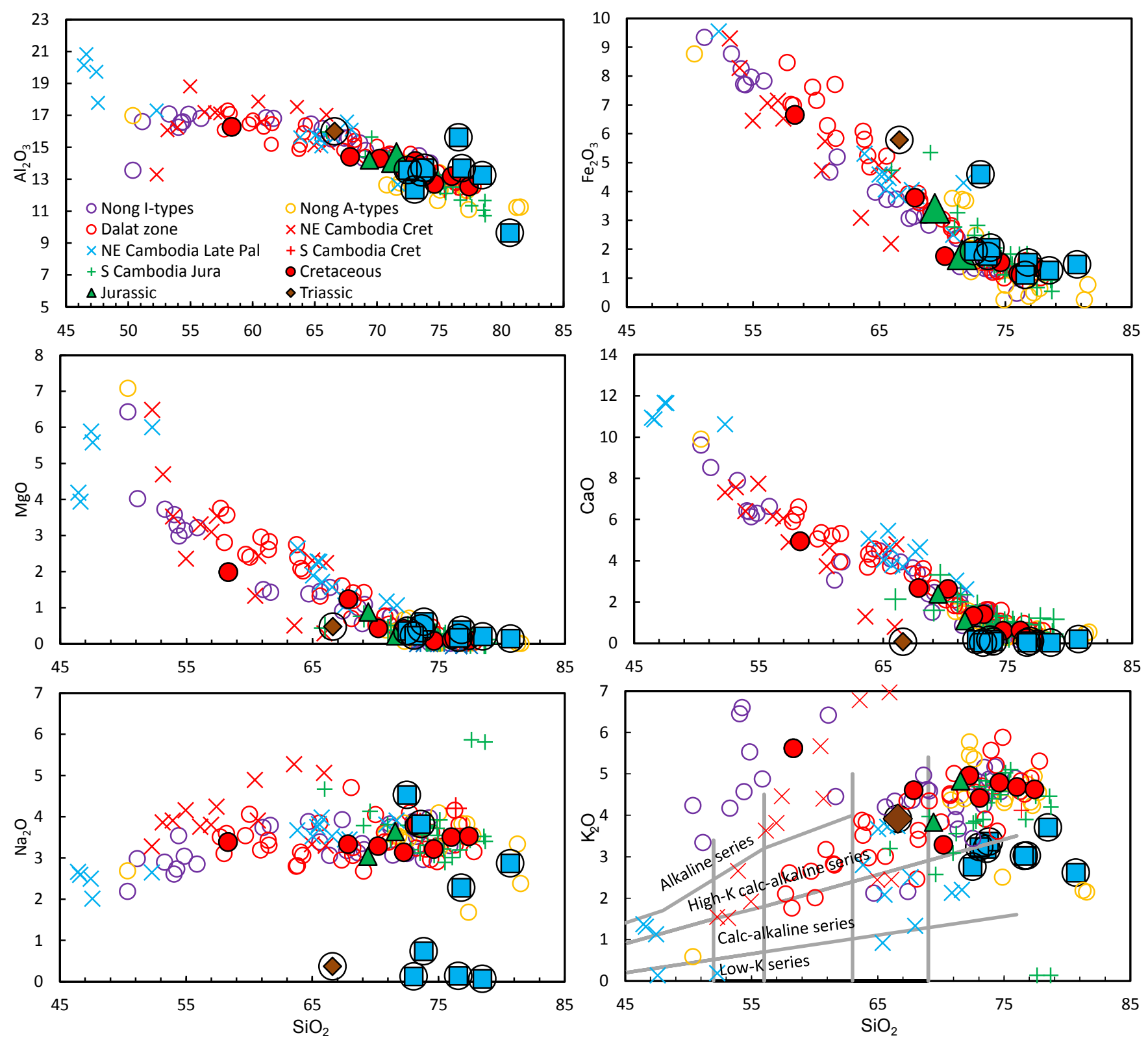

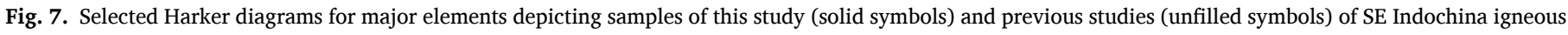
rocks. Classification boundaries in the $\mathrm{SiO}_{2}-\mathrm{K}_{2} \mathrm{O}$ diagram from Ewart (1982), literature data sources given in caption to Fig. 4.

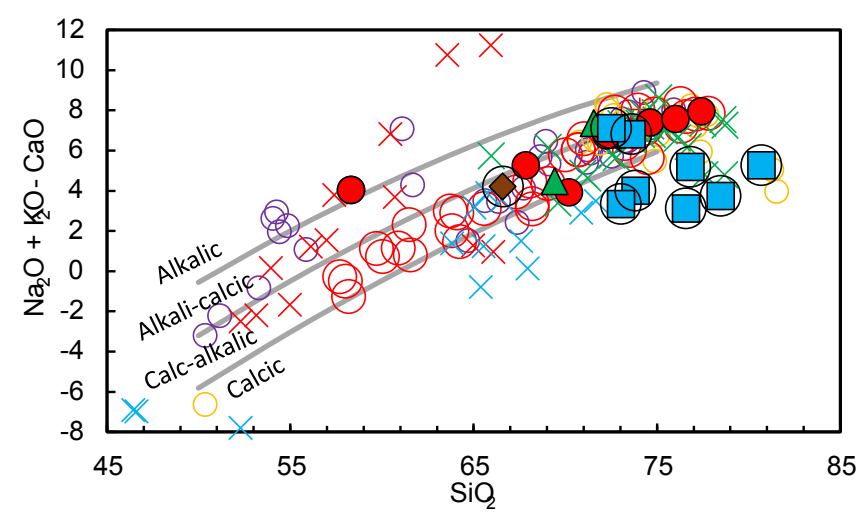

Fig. 8. Modified alkali-lime diagram of Frost et al. (2001). Symbols as in Fig. 4. identified Triassic and Permian rocks are solely located to the west of the transition between the Cretaceous Granite Belt and the Kampot Fold Belt (Fig. 2). It is not clear if this reflects the existence of a significant preCretaceous structural boundary or it merely reflects differential erosion between durable granites and less durable sediments and volcanics. A number of hydrogeological wells in the east encountered preNeogene strata attributed to the same Paleozoic and Triassic formations existing in the Kampot Fold Belt (Hoa, 1996). It is not clear if Permian and Triassic volcanics were encountered in these wells, but the presence of apparently comparable Paleozoic-Triassic lithostratigraphy in both the Granite Belt and the Kampot Fold Belt questions the presence of a major pre-Cretaceous tectonic boundary.

Intense latest Cretaceous-Eocene deformation along the Kampot Fold Belt was suggested as a result of the accretion of Luconia to Indochina and the cessation of Paleo-Pacific subduction (Fyhn et al., 2010a), but contrasts with other Luconia models (Hall, 2012; Morley, 2012). Most of the Cretaceous Granite Belt to the east is concealed underneath Holocene alluvium and exposures are limited to isolated hills speckling the 

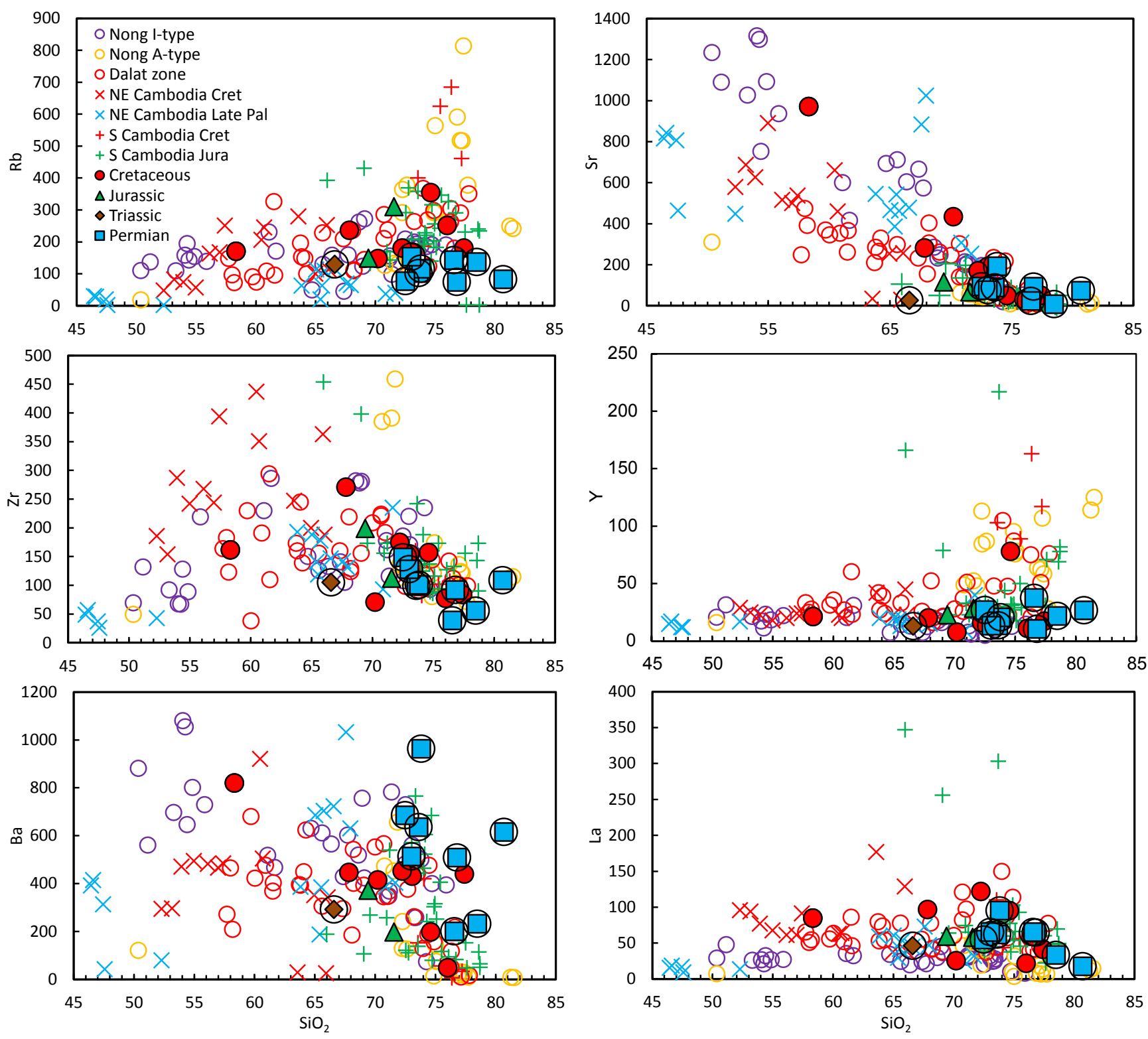

Fig. 9. Selected trace element Harker diagrams. Literature data sources in Fig. 4.

fringe of the Mekong Delta. However, the granitic belt may have formed a block of relatively rigid lithosphere transferring compressional forces from the Paleo-Pacific fringe to the Kampot Fold Belt in the west.

\subsection{Tectonic affiliations}

In the following section, we discuss the tectonic affiliations of the various age groupings identified in this study and link them to the known tectonic evolution of SE Asia. A fundamental uncertainty for defining the tectonic affinity of especially the pre-Cretaceous rocks in S Cambodia and $S$ Vietnam lies in ambiguities around extrapolation of various relatively well-defined major terrane boundaries in Thailand to the SE into Cambodia and Vietnam, and also further south into Malaysia. In particular, the south-eastwards extension of the Sa Kaeo Suture separating the Carboniferous to Permian Sukhothai Arc Terrane from Indochina is largely unconstrained (see Fig. 2). Sone et al. (2012) extrapolated this suture in a south-easterly direction from $\mathrm{E}$ Thailand through S Cambodia and Vietnam, which could suggest affiliation of pre-Cretaceous magmatism in S Cambodia and Vietnam with the Sukhothai Arc and PaleoTethys subduction (see also Sone and Metcalfe, 2008; Zaw et al., 2014;
Qian et al., 2016; Wang et al., 2018). In contrast, Metcalfe (2013a, b, 2021) extrapolates this suture directly south from Thailand (as shown in Fig. 2), thus placing S Cambodia and S Vietnam firmly within the Indochina Block and potentially several hundred km from the site of PaleoTethys subduction (see also Metcalfe, 2017; Faure et al., 2018).

\subsubsection{Cretaceous samples - Tectonic affinities and comparison to the Dalat Zone, NE and $S$ Cambodia}

Cretaceous activity in SE Asia can be confidently linked to westwardsdirected subduction of the Paleo-Pacific Plate beneath Indochina. Investigations of granitic magmatism in southern Vietnam have primarily focused on the Dalat Zone - a region dominated by mid-Late Cretaceous Itype calc-alkaline granitic magmatism. The Dalat Zone represents part of a major Cretaceous subduction zone that stretched from China, through Vietnam, south to Borneo (e.g., Zhou et al., 2006, Hall, 2012; Xu et al., 2016; Breitfeld et al., 2020; Guo et al., 2021) and possibly also extends to the Cretaceous arcs of Australia and New Zealand (e.g., Zahirovic et al., 2016). Four main granitic suites are identified in the Dalat Zone, based primarily on petrographical and textural characteristics (Tran and Vu, 2011). The Dinquan Suite comprises I-type hornblende-bearing diorites 

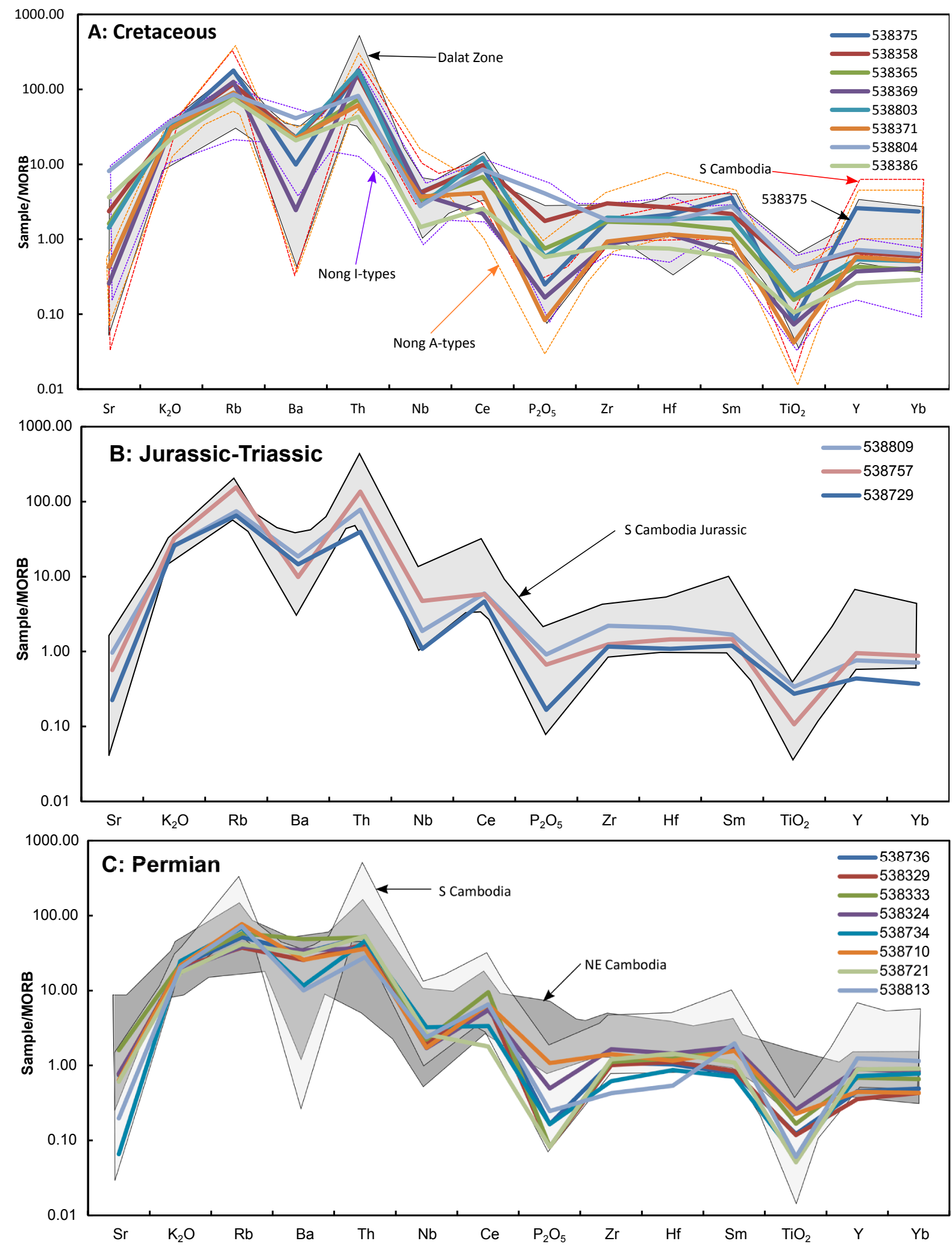

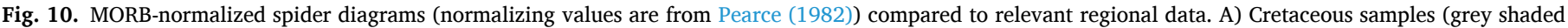

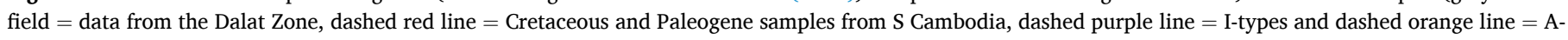

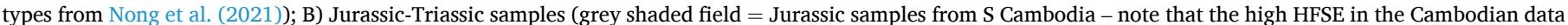

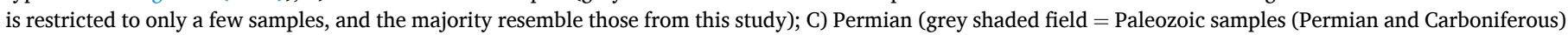

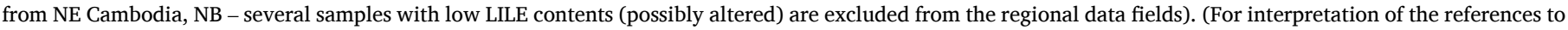
colour in this figure legend, the reader is referred to the web version of this article.) 

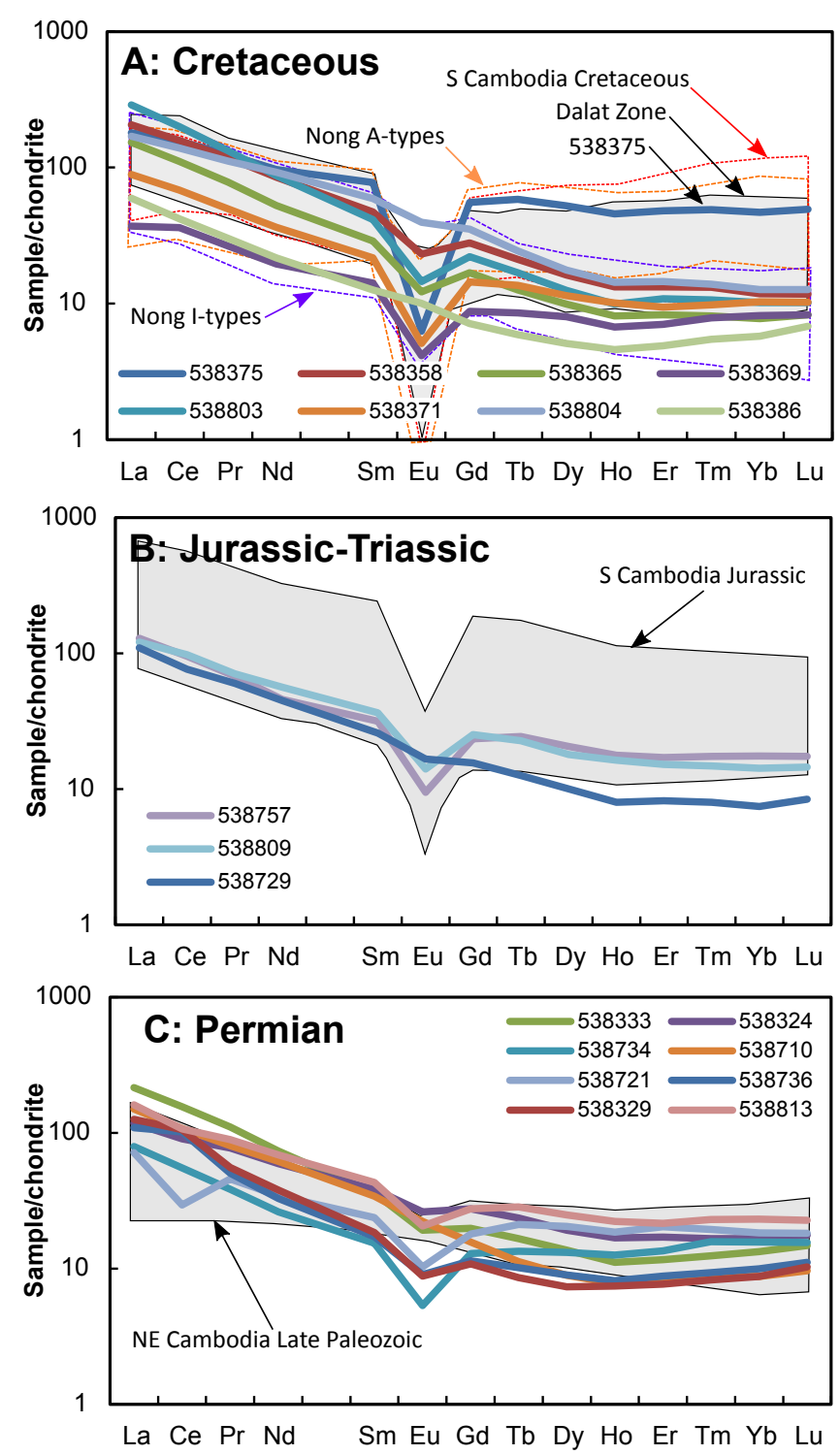

Fig. 11. Chondrite-normalized (Anders and Grevesse, 1989) REE plots for samples of this study compared to relevant literature data. A) Cretaceous samples (Grey field is published data for the various suites of the Dalat Zone, dotted red line is field for Cretaceous and Paleogene samples in S Cambodia, dashed purple line $=$ I-types and dashed orange line $=$ A-types from Nong et al. (2021)); B) Jurassic-Triassic samples (Grey field is published data for the Jurassic samples from S Cambodia); C) Permian samples (Grey field is published data from NE Cambodia). (For interpretation of the references to colour in this figure legend, the reader is referred to the web version of this article.)

to biotite granodiorites with zircon ages of around 112-100 Ma (Nguyen et al., 2004; Shellnutt et al., 2013). The Deoca Suite consists of hornblende and biotite-bearing granodiorites, monzogranites and diorites. Nguyen et al. (2004) provide U-Pb zircon dates for two samples that range between 92 and $91 \mathrm{Ma}$ and suggest an age range of 92-88 Ma, whereas Shellnutt et al. (2013) have dated a third sample of this suite to $118 \mathrm{Ma}$ (this sample has been re-interpreted to belong to the Dinquan Suite, T. Breitfeld pers. comm. 2021 (see also Henning-Breitfeld et al. (2021)). The Cana Suite is located mainly along the SE coast of Vietnam and has been dated to between 96 and 93 Ma (Nguyen et al., 2004; Thuy et al., 2004). Finally, the Ankroet Suite is located mostly in the west of the Dalat Zone, has been dated to around $87 \mathrm{Ma}$, and consists of leucocratic two-mica granites with scarce hornblende and variable amounts of tourmaline (Tran and Vu, 2011; Shellnutt et al., 2013).
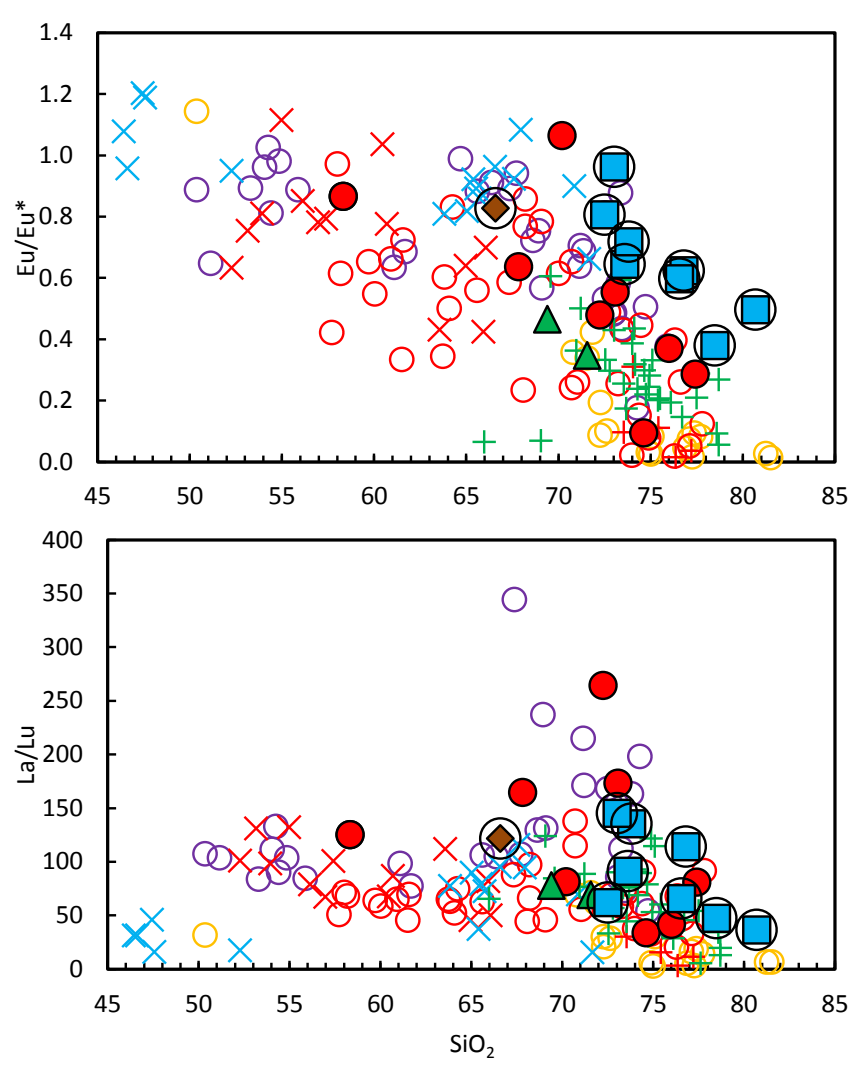

Fig. 12. Plot of $\mathrm{SiO}_{2}$ vs $\mathrm{Eu}$ anomaly and $\mathrm{La} / \mathrm{Lu}$. See Fig. 4 for sources of literature data, symbols as in Fig. 8. A few unusual I-types from Nong et al. (2021) with elevated La/Lu (up to 800) are excluded.
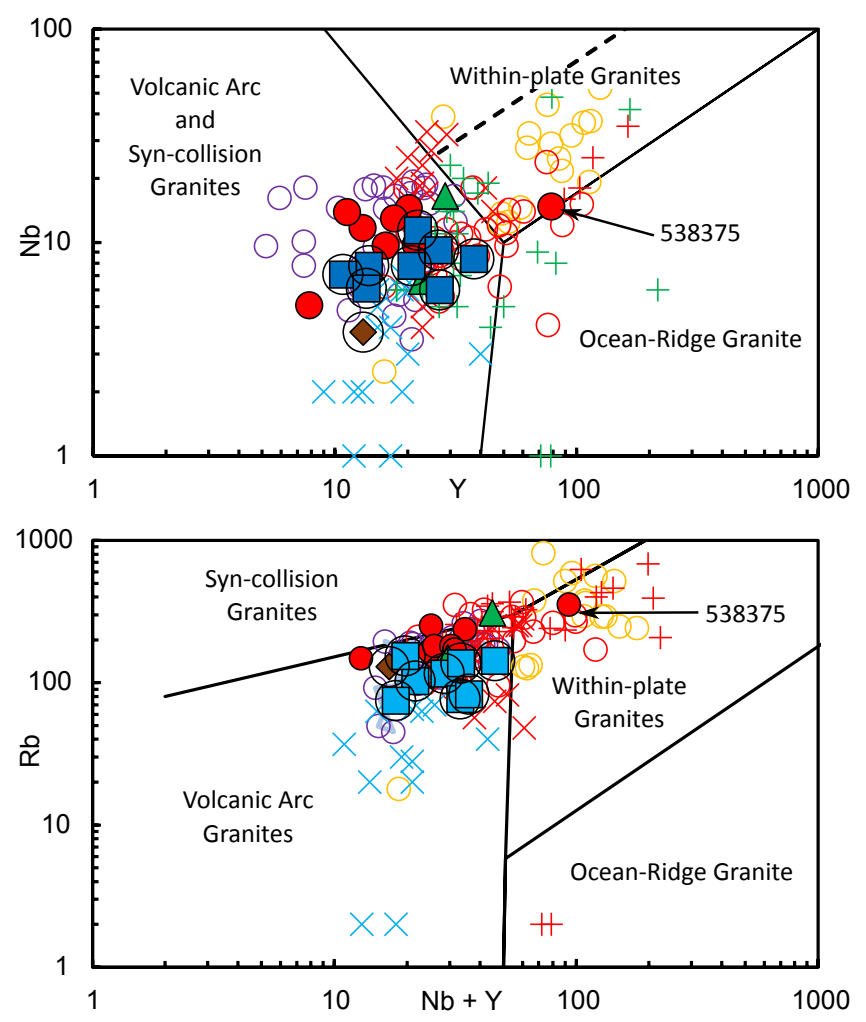

Fig. 13. Tectonic discrimination diagrams from Pearce et al. (1984) (see Fig. 4 for sources of literature data), symbols as in Fig. 9. 
Geochemical differences between the Dalat Zone suites are subtle. The Dinhquan Suite is characterized by generally lower silica contents compared to the other suites, but all suites show mostly coherent trends in most Harker diagrams and similar patterns on mantle-normalized multi-element plots (Shellnutt et al., 2013; Thuy et al., 2004). The aforementioned studies propose that there are differences in isotopic compositions between the various suites, suggesting distinct sources, however when the two data sets are combined it shows that all suites broadly overlap in $\mathrm{Sr}$ and $\mathrm{Nd}$ isotope space with ${ }^{87} \mathrm{Sr} /{ }^{86} \mathrm{Sr}_{(\mathrm{i})}=$ 0.704-0.706 and $\varepsilon \mathrm{Nd}_{(\mathrm{i})}=+1$ to -3 . These compositions are typical for many Cordilleran Arc type settings globally and suggest that old continental crust played a lesser role during petrogenesis. A potentially important contrast is that the Ankroet Suite tends to have higher Y and $\mathrm{M}$-HREE contents at a given silica content, resulting in it plotting in the within plate granite and A-type granite fields in the tectonic discrimination diagrams of Pearce et al. (1984) and Whalen et al. (1987). This led to an overall interpretation that the Dinhquan, Deoca and Cana Suites are I-type granites produced in a continental arc setting (Nguyen et al., 2004; Thuy et al., 2004; Shellnutt et al., 2013), whereas the younger Ankroet Suite formed in a post-orogenic extensional setting (Shellnutt et al., 2013; Henning-Breitfeld et al., 2021).

Recently, Nong et al. (2021) have published U-Pb zircon ages and geochemistry for granitoids west of the Dalat Zone in SW Vietnam and SE Cambodia, investigating a suite of samples that overlap with the eastern portion of our study (east of the transition zone to the Kampot Fold Belt) as well as further east to offshore Vietnam. They recognize several suites of I-type arc-related rocks with ages between 107 and 91 $\mathrm{Ma}$, and that are predominantly calc-alkaline in composition but some also extending to higher $\mathrm{K}$ contents. The most abundant of these I-type rocks belong to the Bay Nui-Phnom Den Suite (ca. 94-91 Ma), which Nong et al. (2021) correlate to the Deoca Suite of the Dalat Zone. Nong et al. (2021) also identify several younger granites (83-75 Ma) with Atype characteristics which they related to extension and the Ankroet Suite of Shellnutt et al. (2013).

The dates of the Cretaceous granitoids investigated in this study (107-75 Ma) as well as those in Nong et al. (2021) overlap with published dates from the Dalat Zone (118-88 Ma) and indicate that the Cretaceous arc continued further south and inboard from the Dalat Zone (Fig. 14). Petrographically, the SW Vietnam and S Cambodian granites also show many similarities to the Dalat Zone rocks, ranging from classic metaluminous amphibole-biotite granodiorites at lower $\mathrm{SiO}_{2}$ contents to weakly peraluminous muscovite-biotite granites at higher silica contents - a typical evolution trend for Cordilleran I-type granites. Furthermore, the majority of the Cretaceous samples identified here were emplaced between 98 and $88 \mathrm{Ma}$ and show similar geochemical trends and compositions to the Dalat Zone rocks (Figs. 7 and 9), with the exception of the youngest sample 538375 (Tonleap, $75 \mathrm{Ma}$ ) and samples 538386 (PhnomAya) and 538804 (Hon Tre S) (both $107 \mathrm{Ma}$ ) which will be discussed separately below. The generally high $\mathrm{SiO}_{2}$ contents $(>67 \%)$ and tectonic classification as volcanic arc granites for the midCretaceous (c. $100 \mathrm{Ma}$ ) samples would suggest affiliation with the Deoca Suite rather than the lower silica Dinqhuan Suite. However, the Late Cretaceous samples studied here are distinguished by lower HREE contents $\left(\mathrm{Lu}_{\mathrm{N}}=6.8-12.6\right)$ than both the Deoca and Dinqhuan Suites $\left(\mathrm{Lu}_{\mathrm{N}}=12.2-23.7\right)$. We suggest that this reflects a greater depth of melting and therefore the presence of residual garnet. This may be a consequence of their more inboard nature and therefore thicker crust and greater depth to the subducted slab.

As mentioned above, three samples are distinct from the other Cretaceous samples. Sample 538804 (107 Ma) is distinguished by relatively low silica contents (58.3 wt $\%$ ), high $\mathrm{K}_{2} \mathrm{O}$ and plotting in the alkali field in the MALI classification plot (Fig. 8). Such compositions are unknown in the Dalat Zone, but are observed in NE Cambodia (Cheng et al., 2019) - for example, in rocks from Kon Mom dated to $103 \mathrm{Ma}$. We note that sample 538804 overlaps with compositions from NE Cambodia in most geochemical plots. However, relating this sample to contemporaneous activity in NE Cambodia is complicated by the clear contrasts in rock compositions across the Mae Ping Fault demonstrated by Cheng et al. (2019). Notably, both sample 538804 and 538386 (Phnom Aya, 107.3 Ma) have similarly shaped REE patterns (lacking significant negative Eu anomalies) and have high $\mathrm{Sr} / \mathrm{Y}$ features commonly attributed to adakitic compositions (Defant and Drummond, 1990). Sample 538386 has a moderate LOI (2.54) which together with petrographic observations suggests some alteration, however its CIA is $<55$ suggesting alteration has not affected the major element compositions. REE should be relatively stable during alteration and we suggest that the lower M-HREE abundances and lack of Eu anomaly in this sample are unlikely to reflect alteration as the sample shows no Ce anomaly, and no disturbances in the LILE. Instead, this distinct REE pattern suggests a primary signature resulting from dominant removal/retention of amphibole (removing MREE) over feldspar (removing Eu) during petrogenesis. Adakitic signatures are typically attributed to melting of young, relatively-warm, subducted oceanic crust followed by interactions between the melt and overlying mantle wedge (e.g. Defant and Drummond, 1990) or high pressure partial melting of thickened or delaminated lower continental crust (e. g. Atherton and Petford, 1993; Gao et al., 2004). Adakitic signatures are present in older (Carboniferous-Permian) magmatic rocks in $\mathrm{N}$ Cambodia (Cheng et al., 2019) but have not been previously recognized in the Dalat Zone. However, we note that a single undated sample in the study of Thuy et al. (2004) (DQ12) has a similar REE pattern and high Sr/Y (25). The two samples with adakite-like signatures identified here (538386 (Phnom Aya), 538804 (Hon Tre S)) are older (107 Ma) than the remaining Cretaceous granites in S Vietnam (98-75 Ma), and we propose that they represent a distinct event and suite previously unrecognized in the Dalat Zone. Interestingly, we note that several samples from the Bay Nui Ba Den suite (107-96 Ma) and the offshore Bach Ho suite (110 Ma) of Nong et al. (2021) also have high $\mathrm{Sr} / \mathrm{Y}$, suggesting the adakitic signature may be more widespread in the region.

Sample 538375 (Tonleap) is considerably younger than the other samples studied here (75 Ma) and is geochemically distinguished by much higher HREE contents (Fig. 11), classifying as a WPG/A-type granite in various tectonic discrimination diagrams. This rock correlates with several Late Cretaceous suites of A-type granites in recognized SW Vietnam, including the the Ankroet Suite in the Dalat Zone (87 Ma, Shellnutt et al. (2013), and the Con Dao (83 Ma), Pha Aok (78 Ma) and Nui Sam-Angkor Borei (75 Ma) Suites of Nong et al. (2021). Shellnutt et al. (2013) and Nong et al. (2021) suggested that these A-type magmas are related to post-orogenic collapse following the end of Cordilleran Arc activity. The timing and cause of the cessation of subduction is unclear, but it must have stopped prior to the deformation and exhumation of the Kampot Fold Belt attributed to the collision of Luconia in the latest Cretaceous-Eocene (Fyhn et al., 2010a). A-type granites with similar ages have been identified in southern Borneo (85-72 Ma, Breitfeld et al., 2020) and on the eastern Malay Peninsula (Searle et al. 2012; Metcalfe, 2013a). An even younger post-orogenic A-type granite has also been identified in S Cambodia east of the transition zone (Phnom Da at $58 \mathrm{Ma}$ ) by Cheng et al. (2019). Combined, these rocks suggest a dispersed yet widespread episode of post-orogenic granitic magmatism across SW Asia. Finally, abundant A-type granites are also present to the north in China but these are older (180-140 Ma) and represent an extensional event that either pre-dates continental arc magmatism (e.g. Zhou et al., 2006) or foundering of subducted lithosphere following flat-slab subduction (Li and Li, 2007).

\subsubsection{Jurassic and Triassic samples: Paleo-Tethys or Paleo-Pacific subduction?}

Two Jurassic granites are identified, and have very similar ages (194 Ma) despite a large geographic separation. Both samples are relatively high silica $\left(\mathrm{SiO}_{2}=70-72 \%\right)$, peraluminous biotite granites that plot in the volcanic arc field in tectonic discrimination diagrams (Fig. 13), and 

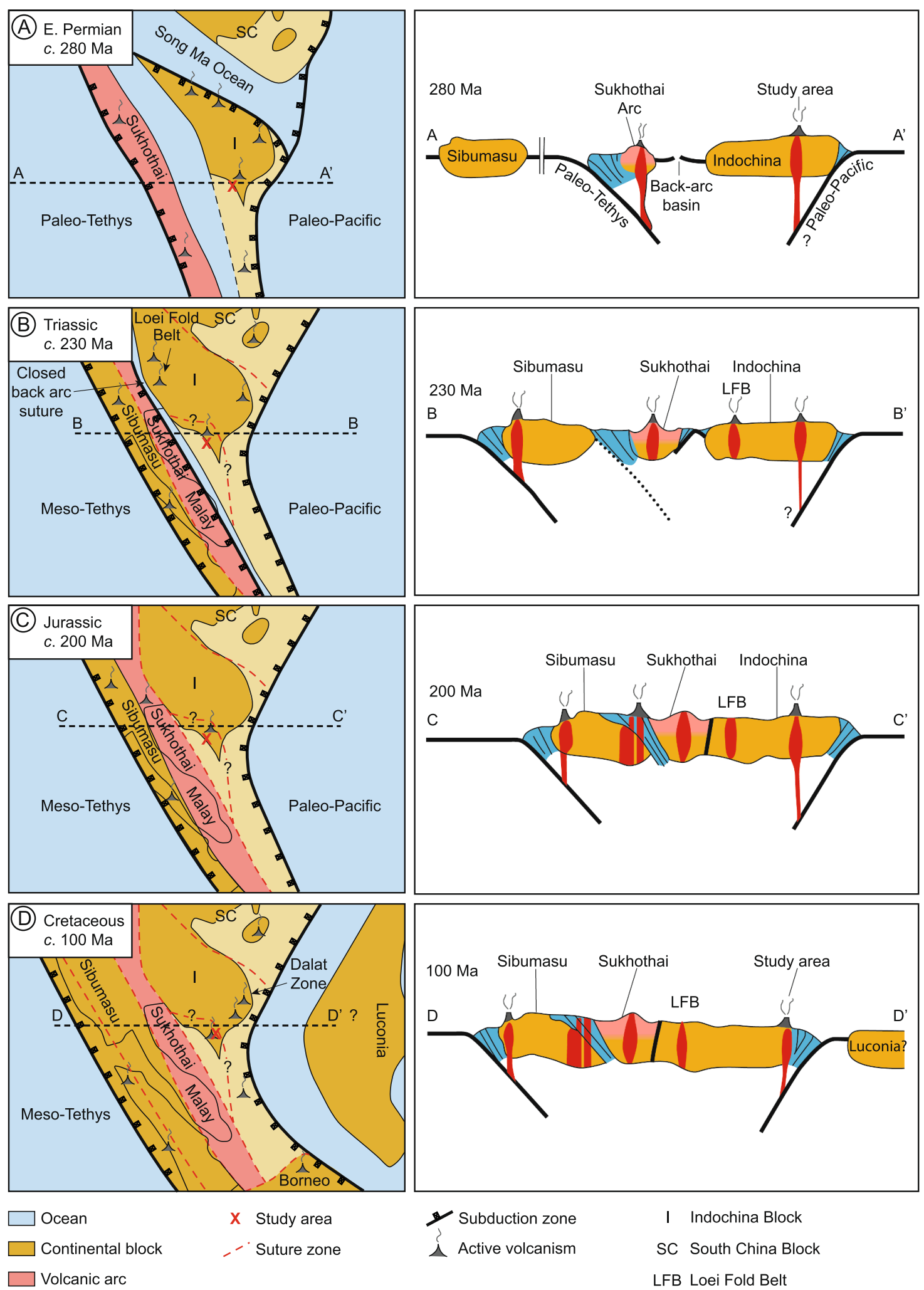

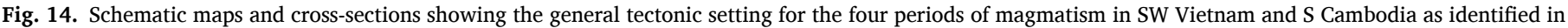

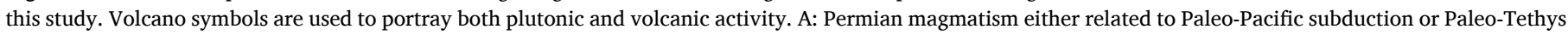

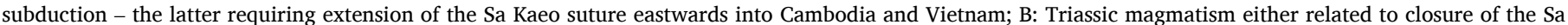

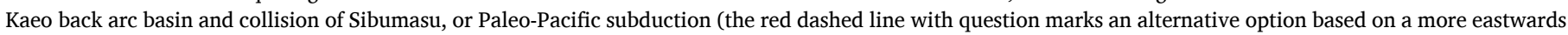

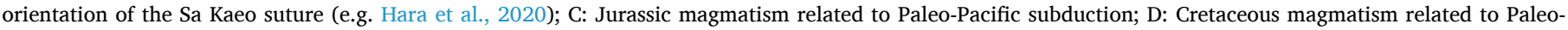

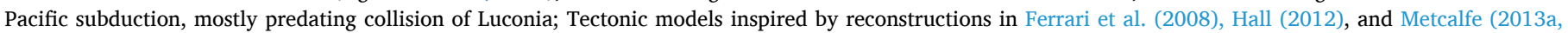
b, 2017). See text for discussion. (For interpretation of the references to colour in this figure legend, the reader is referred to the web version of this article.) 
have trace element abundances typical of many subduction-related rocks (enriched LILE, depletions in Nb, P, Ti). Reliable Jurassic ages are unknown in the Dalat Zone or the more northern Kontum Massif (Faure et al., 2018). However, several granites from S Cambodia (west of the transition zone) have broadly similar ages ranging from 205 to 167 Ma (Cheng et al., 2019), and have generally similar geochemical characteristics (with the exception of a few samples with elevated REE contents). Although the ages of Cheng et al. (2019) are based on whole rock $\mathrm{Rb}-\mathrm{Sr}$ isochrons sometimes derived using samples from widely dispersed intrusions that have been shown here to have different ages, and have comparatively large errors, they suggest that Late Triassic to Early Jurassic granites may be more widespread in the region.

Cheng et al. (2019) attribute Late Triassic-Jurassic magmatism in S Cambodia to E-NE dipping subduction of the Paleo-Tethys beneath SW Indochina, and more specifically suggest correlation of the S Cambodian rocks to granites in the Loei Fold Belt/ Phetchabun Volcanic Belt in NE Thailand (e.g. Nualkhao et al. 2018). These granites are broadly linked to PaleoTethys subduction, and closure of the Sukhothai Back-arc basin along the Sa Kaeo Suture and subsequent outboard collision of Sibumasu. The Jurassic samples from S Cambodia analyzed by Cheng et al. (2019) have ${ }^{87} \mathrm{Sr} /{ }^{86} \mathrm{Sr}_{(\mathrm{i})}$ of around $0.705-0.707$ and ${ }^{143} \mathrm{Nd} /{ }^{144} \mathrm{Nd}_{(\mathrm{i})}$ of $\mathrm{c}$. $0.5123-0.5125$, indicating that they are not juvenile mantle-derived arc compositions, but also that their sources are not dominated by ancient continental crustal components. These isotope compositions are also broadly similar to the more clearly subduction-related Cretaceous granites of the Dalat Zone and suggest derivation from similar crustal sources.

Directly linking Jurassic magmatism to Paleo-Tethys subduction and terrane accretion along the western margin of Indochina is complicated by tectonic models presented by for example Sone and Metcalfe (2008) and Metcalfe (2013b, 2017) that suggest that such collisional events largely predate Jurassic rocks in S Cambodia and Vietnam. These models suggest that by around $230 \mathrm{Ma}$, the main locus of subduction along the western margin of Indochina had moved outboard and involved subduction of Meso-Tethys along the outer margin of the Sibumasu Terrane and was thus on the order of $300 \mathrm{~km}$ west of S Cambodia and SW Vietnam. Closure of the back arc between the Sukhothai Arc and Indochina, and docking of the Sibumasu Terrane to Sukhothai, took place in the midTriassic and thus significantly pre-dates Jurassic magmatism in $\mathrm{S}$ Cambodia and Vietnam by c. 50 Ma. We therefore argue that it is unlikely that these Jurassic rocks are related to subduction-related processes along the western margin of Sundaland, and that they are more likely related to westwards-dipping subduction of the Paleo-Pacific (Fig. 14c).

It is unclear when westwards-dipping subduction of the Paleo-Pacific initiated along the eastern margin of Indochina. Metcalfe (2013b, 2017) provides a large-scale tectonic reconstruction at the Triassic/Jurassic boundary that indicates subduction on both the eastern and western margins of the core of SE Asia (Sundaland). Subduction of Meso-Tethys was occurring along the SW boundary and separated from Indochina by both the Sibumasu Terrane and the accreted Sukhothai Arc. Westwards subduction of the Paleo-Pacific plate is similarly indicated along the opposite eastern Sundaland margin (Metcalfe, 2017). This is in line with large-scale full-fit plate reconstructions of the Triassic and Late Jurassic that portray the eastern Sundaland margin as a long-lasting active convergent margin. This margin extended from China in the north possible as far south as Borneo, and was active from the Permian through to the Late Cretaceous, probably interspersed with periods of back-arc extension and possibly transform faulting (Ferrari et al., 2008; Hall, 2012; Zahirovic et al., 2016). Magmatic products potentially also related to Paleo-Pacific subduction are identified in both S China (e.g. Zhou et al., 2006; Li and Li, 2007; Xu et al., 2017) and Borneo (Breitfeld et al., 2017; Hennig et al., 2017; Burton-Johnson et al., 2020), although in both cases activity is not continuous and periods of magmatic quiescence are identified.

Given their geographical proximity to the Cretaceous arc, we suggest that the Jurassic samples identified in this study, and by inference the $S$ Cambodian granitic samples of Cheng et al. (2019) are most likely related to westwards-dipping subduction of the Paleo-Pacific plate beneath Indochina. If this is the case, the subsequent eastwards migration of magmatic activity in Vietnam from the Jurassic into the Cretaceous is similar to that observed in S China (Zhou et al., 2006).

The affinity of the two Late Triassic samples (538729-222 Ma, $538,811-230 \mathrm{Ma}$ ) identified in this study is enigmatic, and corresponding ages are not identified in Cambodia by Cheng et al. (2019). Both samples are altered, and a geochemical analysis is only available for one of these samples (538729, Phnom Changek) and it has trace element characteristics that are similar to the Jurassic and most Cretaceous samples. Most reconstructions from around this time show that SW Vietnam and S Cambodia are significant distances from convergent margins on both the eastern and western edges of Indochina (Fig. 14b). For example, Metcalfe (2017) shows that around this time the back arc between the Sukhothai Arc and Indochina on its western margin was closed or closing. Similarly, magmatic activity in the Sukhothai Arc was waning, and the outboard Sibumasu Terrane was in the process of colliding with the Sukhothai Arc. Granitoids with similar ages (222-218 $\mathrm{Ma}$ ) are found within the Chanthaburi Terrane (Sukhothai equivalent) in SE Thailand (Sone et al., 2012; Qian et al., 2017) and could represent plutonic equivalents to the two Triassic volcanic rocks identified here. These Triassic rocks could also be correlated to rhyolitic volcanics of the Lampang Volcanic Belt within the Sukhothai Arc and located west of the Sa Kaeo suture in eastern Thailand (Ko Chang and Maenum Bang Pakong areas) suggested to be Triassic to mid-Triassic in age (242-237 Ma, Barr et al., 2000; Barr and Charusiri, 2011; Sone et al., 2012; Jiang et al., 2021). U-Pb zircon dates of 240-233 Ma were also obtained for rhyolites in northern Thailand (Sukhothai Terrane) (Barr et al., 2000, 2006), and similar ages are found in the Eastern Granite Province in the southern extension of the Sukhothai Terrane on the Malay Peninsula (Sone and Metcalfe, 2008; Searle et al., 2012; Metcalfe, 2013a; Ng et al. 2015). Correlations with these rocks would be consistent with a southeastwards extension of the Sa Kaeo Suture Zone into S Cambodia and Vietnam as postulated by for example Sone and Metcalfe (2008) and Wang et al. (2018).

Alternatively, the Triassic ages identified here could be linked to magmatism in the Loei Fold Belt, as proposed for the younger Jurassic samples by Cheng et al. (2019) (Fig. 14b). Similar ages (252-219 Ma) are obtained for rocks from the Loei Phetchabun volcanics and volcaniclastics from the Luang Prabang Basin in Laos (Qian et al., 2016; Rossignol et al., 2016), although some older radiometric and stratigraphic ages also occur (Barr and Charusiri, 2011). These volcanics occur east of the Sukhothai Terrane in the Simao subterrane, and extend from Laos, through Thailand potentially into Cambodia (Qian et al., 2016) and were used by Rossignol et al. (2016) to infer eastwardsdipping subduction beneath western Indochina throughout most of the Triassic. Younger magmatism in the Loei Fold Belt (240-206 Ma) overlapping with the Triassic ages in this study has also been attributed to crustal thickening and/or collapse following arc accretion (Jiang et al., 2021). Affiliation to either the Sukhothai Arc or the Loei Fold Belt is dependent on the location of the Sa Kaeo suture zone - which is unresolved in our study area (Also see discussion in 5.2.3). Recent studies have identified Early Permian felsic rocks of the Pailin Igneous Complex in W Cambodia as an eastwards extension of the Nan back arc and Sa Kaeo suture into W Cambodia (Hara et al., 2020). Furthermore, these authors and Nguyen and Nguyen (2017) suggest that gabbros and ultramafic rocks to the east near Ho Chi Minh may represent an extension of this structure further east into Southern Vietnam.

Wang et al. (2018) provide a comprehensive summary of Devonian to Triassic ages from most of the western portion of SE Asia (see Fig. 14). They identify a peak in the dates at around $220 \mathrm{Ma}$, which overlaps with the two Triassic samples of this study and which they attribute to magmatism associated with post-collisional gravitational collapse of the orogenic belt formed by earlier collision of several continental fragments (including Sibumasu) with Indochina. 
Finally, we also note that we cannot exclude that the limited Triassic magmatic rocks identified in S Cambodia and Vietnam were related to Paleo-Pacific subduction along the eastern margin of Indochina (Fig. 14b), which was also ongoing in the Triassic (Ferrari et al., 2008; Hall, 2012; Zahirovic et al., 2016; Wang et al., 2018).

\subsubsection{Permian magmatism}

The high silica contents and altered nature of the Permian samples identified here hinder their petrogenetic interpretation. Broadly speaking they show classic subduction-like trace element signatures with depletions of $\mathrm{Nb}, \mathrm{Ba}, \mathrm{P}_{2} \mathrm{O}_{5}$ and $\mathrm{TiO}_{2}$, and plot in the volcanic arc field in various tectonic discrimination diagrams (Figs. 10 and 13). Compared to the younger samples from the region, they appear to have relatively high $\mathrm{Ba}$ and relatively low $\mathrm{Rb}$ (Fig. 9), which may reflect the mobility of these elements during alteration as they also clearly show sign of loss of Ca and $\mathrm{K}$ (Fig. 7). The relatively high Ba may however be a distinct source and/or petrogenetic signature. The Permian samples have similar REE patterns that show flat HREE and slight depletions of the MREE (lows around Dy) that suggest removal of amphibole (Fig. 11). The Permian samples are also characterized by less distinct negative Eu anomalies at a given silica content compared to younger samples (Fig. 12) which indicate lesser degrees of involvement of feldspar during petrogenesis. The geochemical signature and the closely spaced dates of the Early Permian magmatic rocks investigated in this study suggest a period of active subduction next to S Cambodia and SW Vietnam between at least 294 and 265 Ma. Even though subduction is reasonably well-constrained by our data, the location of the subduction zone is not.

Most discussions of the tectonic setting and associated magmatism in SE Asia in the Permian focus on subduction of Paleo-Tethys along the modern-day western margin of Indochina (e.g., Metcalfe (2017) and references therein). Permian subduction of Paleo-Tethys along the current western margin of Indochina resulted in formation of the Sukhothai Arc, which is correlated with the Chanthaburi Terrane in Thailand and the Eastern Province on the Malay Peninsula (e.g. Metcalfe 2013b, 2017). Initiation of this arc is poorly constrained, but may have been as early as lower Carboniferous based on the presence of marine sediments and volcaniclastics of this age in the Chanthaburi Terrane. Activity in the arc continued until at least the Late Triassic, and ceased with the accretion of the Gondwana-derived Sibumasu Terrane in the mid-Late Triassic (Metcalfe, 2013b, 2017).

The Permian dates in this study (294-265 Ma) overlap broadly with activity in the Sukhothai-Chanthaburi arc and thus could suggest correlation with this activity. However, in detail our dates appear to be somewhat older than potential equivalents in the Sukhothai-Chanthaburi arc. Granites in the E Thailand region of the Chanthaburi Terrane are considerably younger (195-207 Ma) than the Permian volcanics in S Cambodia and SW Vietnam (Sone et al., 2012). These authors also show that Early Permian igneous rocks are presumably lacking in the Chanthaburi Terrane. This contrasts with S Cambodia and SW Vietnam where Early Permian igneous rocks occur in abundance. A direct correlation between the Chanthaburi Terrane and S Cambodia and SW Vietnam is, therefore, not immediately apparent based on volcano-stratigraphic considerations. However, Sone et al. (2012) do discuss a series of volcaniclastic sediments in the southern part of the Sukhothai Terrane (the Dan Lan Hoi Group and the Thung Saliam Group) which contain a number of pyroclastic units that are suggested to be Early Permian (based on fossil evidence) and therefore potentially overlap with the Permian volcanics in S Cambodia and SW Vietnam. However, to our knowledge, more detailed geochronological and geochemical data have not been published for these rocks.

Similarly, dates of granites from the central and eastern belts of the Malay Peninsula (southern correlative of Sukhothai-Chanthaburi) are generally younger than the Permian rocks identified here in our study area. Even so, some older dates (285-260 Ma) are known from Singapore and surrounding areas (Oliver et al., 2014; Ng et al., 2015; Gillespie et al., 2019) as well as in Laos and N Vietnam next to the Song Ma suture.
Wang et al. (2018) review Devonian to Triassic magmatic ages in the western portion of SE Asia related to eastwards subduction of Tethys. More specifically, data from two sutures (Jinghong-Nan-Uttaradit and Luang Prabang-Loei-Phetchabun (see Fig. 1)) in their compilation are relevant for both the Permian and Triassic samples of S Cambodia and SW Vietnam. The Jinghong-Nan-Uttraradit suture represents the boundary between the Sukhothai Terrane and Indochina, and the Luang Prabang-Loei-Phetchabun suture represents the boundary between crustal blocks within Indochina itself. The Jinghong-Nan-Uttaradit suture extrapolates to the south to the Sa Kaeo suture, whereas the LoeiPhetchabun extends south to Thailand but not further into Cambodia or Vietnam. Fig. 15 shows the age ranges of the Permian and Triassic samples identified in this study compared to the age compilation for western SE Asia of Wang et al. (2018) (their Fig. 13f). In particular, it is noteworthy that the Permian dates identified here (260-294 Ma) are comparatively rare around these sutures and fall within the period they define as being dominated by arc/back arc magmatism. Some Early Permian ages are identified in the Inthanon-Bentong Raub suture (references in Wang et al., 2018). However, this is the main suture between the Sukhothai Terrane and the more outboard Sibumasu Terrane (see Fig. 1), and we consider these lithologies to be too distal to be related to activity in S Cambodia and SW Vietnam.

A number of additional issues complicate direct correlation of the Permian volcanics in our study area with the Sukhothai-Chanthaburi Arc. This arc is separated from Indochina by the Nan-Uttaradit - Sa Kaeo suture, which is considered to represent the remnants of a back arc basin that opened between the Sukhothai Terrane and Indochina in at least the Early Permian (e.g. Metcalfe, 2017; Hara et al., 2018). Correlation of the Permian volcanics in S Cambodia and SW Vietnam with the Sukhothai Arc would require extension of the Sa Kaeo suture zone east of Thailand into S Cambodia and SW Vietnam, and as noted above this has been tentatively suggested in several (but not all) reconstructions (e.g., Sone et al., 2012; Zaw et al., 2014; Qian et al., 2016; Wang et al., 2018; Hara et al., 2020). Such an extrapolation is at a significant angle to other major structures in the region that typically have more N-S or NW-SE directed trends. A prominent, roughly N-S trending compressional belt exist underneath a thick Cenozoic cover in the central Gulf of Thailand (Fyhn et al. 2010a). The orientation and the location of the belt made Fyhn et al. (2010a) interpret it as the offshore extension of the Chanthaburi deformation belt and the eastern fringe of the Chanthaburi Terrane. Many other reconstructions similarly extrapolate the Sa Kaeo suture south of Thailand (e.g. Sone and Metcalfe 2008; Sone et al. 2012; Zaw et al. 2014; Qian et al. 2016; Wang et al. 2018) placing the Permian volcanics of S Cambodia and SW Vietnam clearly within Indochina.

The timing of opening of the Nan-Uttaradit - Sa Kaeo back arc basin is unclear. Reconstructions in Metcalfe (2013b, 2017) suggest it opened at some point between 299 and 270 Ma (see also Hara et al., 2018), although Late Carboniferous MORB gabbros and basalts in the NanUttaradit suture (335-304 Ma) suggest it was open earlier at least in the north (Qian et al., 2016; Yang et al., 2016; Wang et al., 2018). In addition, identification of Lower Permian (Asselian to Sakmarian) radiolarians and conodonts in chert in the Chanthaburi area suggests relatively deep marine conditions already existed in the back arc by this time (Saesaengseerung et al., 2009). If the Permian volcanics of S Cambodia and SW Vietnam are related to the Sukhothai-Chanthaburi Arc, this would then suggest that a significant back arc was present between them and mainland Indochina located to the east. Clearly, more accurate location of this suture zone is essential to our understanding of SE Asian geology, but this is hampered by a lack of outcrop on land, and burial of pre-Cretaceous geology by many kilometers of Cenozoic and Lower Cretaceous sediment in the Gulf of Thailand (Fyhn et al., 2010a,b, 2016).

Another complication for linking the Permian volcanics in $S$ Cambodia and SW Vietnam with the Chanthaburi Terrane in Thailand is that there seems to be a clear contrast in stratigraphy in the two regions. $\mathrm{S}$ Cambodia is dominated by lower Paleozoic marine sediments (Cambrian to Devonian siliciclastics) overlain by Carboniferous, 


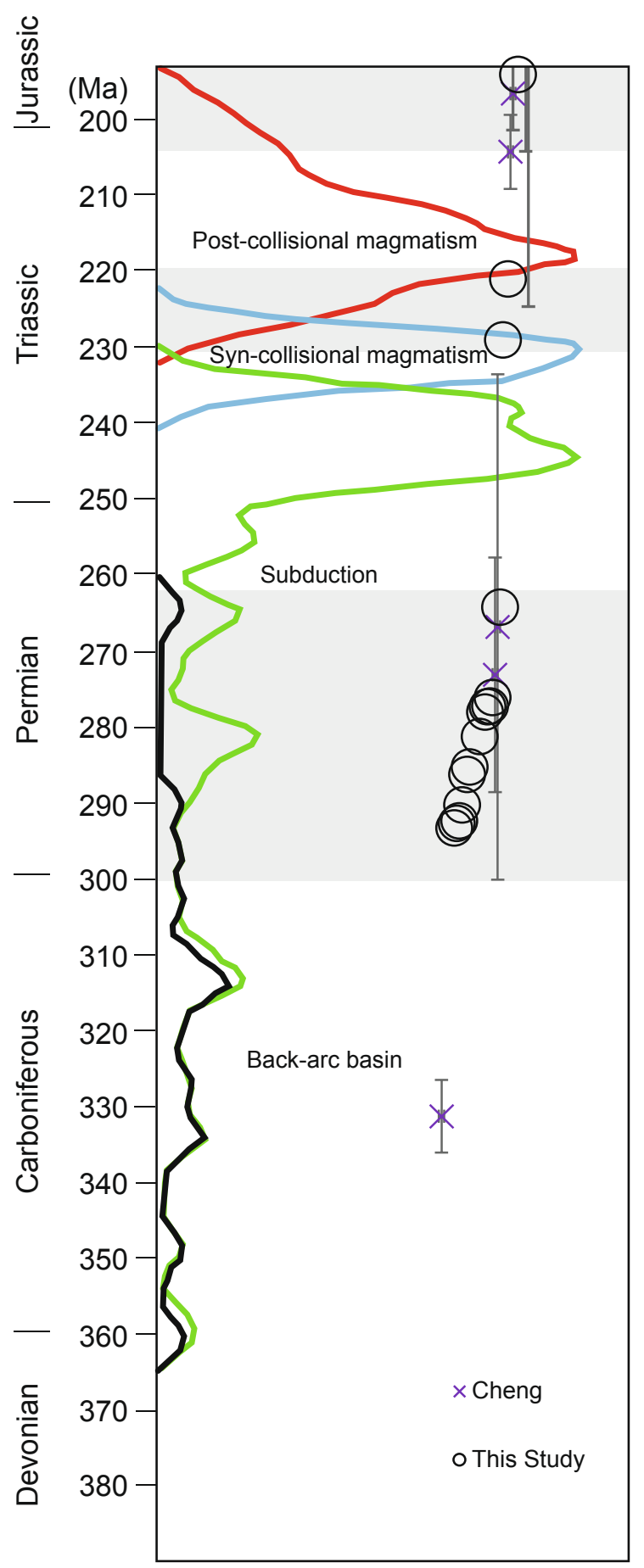

Fig. 15. Comparison of published and new pre-Cretaceous ages obtained in this study from SW Vietnam and S Cambodia (Cheng = Cheng et al. (2019)) with ages compiled from the southwest margin of SE Asia by Wang et al. (2018) (their Fig. 13 F). Black = Back arc magmatism, Green = subduction, Blue = syncollision, Red = post collision - the grey boxes show the age distributions of the Permian, Triassic and Jurassic samples identified in this study. Note that the ages from S Cambodia do not correlate well with the magmatic history from SW SE Asia as identified by Wang et al. (2018). Error bars on most analyses (U-Pb zircon) are smaller than symbol size. (For interpretation of the references to colour in this figure legend, the reader is referred to the web version of this article.)
Permian and Triassic siliciclastics, carbonates and volcaniclastics in many respects comparable with the stratigraphy within the central Indochina Block (Booth and Sattayarak, 2011; Fyhn et al., 2016; Tran and $\mathrm{Vu}, 2011)$. In contrast, pre-Carboniferous sediments are not known in the Sukhothai or Chanthaburi Arcs, which are dominated by Permian to Triassic marine siliciclastics, carbonates and volcaniclastics (Sone et al., 2012).

As an alternative to eastwards-dipping subduction, the Permian volcanics could also be related to rocks with broadly similar ages from the Jinshajiang-Ailoshan-Song Ma and Troung Son sutures in S China and N Vietnam (see Fig. 1) along the north-eastern fringe of the Indochina Block, (e.g. Thanh et al., 2019). These igneous rocks are likely related to south-westwards-dipping subduction of the Song Ma Ocean underneath the northern Indochina Block along the Song Ma subduction zone (Thanh et al. 2019, Tran et al., 2020). It is uncertain how the Song Ma subduction zone linked with the Paleo-Pacific trench system in the Early Permian, but from geodynamic considerations a link seems most likely. Fig. 14a proposes a link between the two subduction systems and a distant link with the Permian magmatic activity in $S$ Cambodia and SW Vietnam.

As an alternative to correlating the Permian volcanics with eastwards Paleo-Tethys subduction and the Sukhothai Arc or southwards subduction of the Song Ma ocean, we instead suggest that an alternative explanation is to link them to westwards-dipping subduction of what we refer to as the Paleo-Pacific Plate located to the east of the study area. In detail, distinction between Paleo-Tethys and Paleo-Pacific subduction in the Permian is blurred as they may represent a single integrated system (e.g., Ferrari et al., 2008). Most research articles focus on subduction along the current western side of Indochina, likely because of better accessibility and exposure, and the role of subduction along the eastern margin is often overlooked. Several reconstructions indicate westwardsoriented subduction of the Paleo-Pacific was occurring or initiated along the eastern margin of Indochina in the Permian simultaneous with formation of the Sukhothai Arc, and these reconstructions typically show Indochina as a 'peninsular' surrounded by subduction zones (e.g., Ferrari et al., 2008; Metcalfe, 2013b, 2017). The various Early Permian reconstructions of Metcalfe (2013a, b, 2017) are large-scale and thus identifying how the various subduction zones relate in detail to $S$ Cambodia and SW Vietnam is unclear, but show subduction extending from the modern-day western Indochina, to western Sumatra, and further to along the margins of both southern and northern China. In the more detailed 290 Ma reconstruction of Ferrari et al. (2008), subduction is shown along the entire eastern side of Indochina, extending north to South China, and south to western Sumatra and eastern Gondwana (Australia). Ferrari et al. (2008) however, do not show subduction along the western margin of Indochina (their Shan Thai Block that includes the Sukhothai Arc) and instead show southwards-dipping subduction along a distal Sibumasu Terrane - an interpretation Metcalfe (2009) finds unconvincing. Permian magmatism in SE Asia related to subduction along its eastern margin is poorly documented, and the onset of PaleoPacific subduction is the subject of debate. Notably, several authors have invoked Early to Mid Permian subduction to explain granitic magmatism and detrital zircon populations in S China (e.g., Li et al., 2006; Li and Li, 2007; Hu et al., 2015). Permian magmatic activity related to Paleo-Pacific subduction is well documented in Australia (New England Batholith e.g., Cawood et al., 2011) and New Zealand (Brook Street Terrane e.g., Spandler et al., 2005).

In summary, a link between Early Permian magmatism in $S$ Cambodia / SW Vietnam and Paleo-Pacific subduction from the east fits with the, admittedly, scarce but consistent evidence for Pacific subduction, and further seems more plausible than the alternative link with Paleo-Tethys subduction system in the west. We therefore tentatively 
suggest that the Permian volcanics in S Cambodia and SW Vietnam could be related to westwards subduction of the Paleo-Pacific.

\section{Conclusions}

Four main age groupings of felsic volcanics and plutonic rocks are identified in SW Vietnam and S Cambodia: Cretaceous (107-75 Ma, $\mathrm{n}=$ 12), Jurassic (195 Ma, $n=2$ ), Triassic (230-222 Ma, $n=2)$, and Early Permian (294-266 Ma, $\mathrm{n}=12$ ). The Cretaceous rocks are restricted to east of the Kampot Fold Belt, whereas the pre-Cretaceous rocks occur further west in the Kampot Fold Belt.

A single relatively young (75 Ma) A-type/within plate granite is recognized and provides increased evidence for a dispersed postorogenic igneous event in the region which broadly overlaps with the cessation of subduction and is possibly linked with the docking of Luconia causing regional deformation and uplift. The majority of the Cretaceous samples formed between 98 and $89 \mathrm{Ma}$ and are related to Paleo-Pacific subduction and the Mesozoic arc system that dominates most of coastal Vietnam. They are distinguished from granites of the Deoca suite by lower HREE contents that may indicate generation deeper in the crust in the presence of garnet. Two granitic samples have adakite-like signatures and may represent a new suite previously unrecognized in Vietnam.

Two granites with Jurassic (195 Ma) ages are identified within or east of the transition zone between the Cretaceous arc and the Kampot Fold Belt. Other studies suggests lithologies of this age are more widespread in the region. These rocks are interpreted as products of westwards subduction of the PaleoPacific beneath Indochina. The affinity of two Triassic samples (222-228 Ma) in the Kampot Fold Belt is unclear and they are tentatively linked to the Loei Fold Belt and attributed to crustal thickening associated with back-arc closure and accretion of the Sibumasu Terrane during eastwards-oriented subduction of Tethys.

Permian (294-265 Ma) rocks are restricted to the Kampot Fold Belt and are mostly altered felsic volcanics and associated granites. Trace elemental systematics are consistent with a subduction-related origin, however their tectonic affinity is unclear. These rocks could potentially be correlated with the Sukhothai and Chanthaburi Terrane volcanics formed by eastwards subduction of Paleo-Tethys. However, this interpretation requires extrapolation of the Sa Kaeo back arc suture eastwards through S Cambodia and S Vietnam and against the dominantly $\mathrm{N}-\mathrm{S}$ structural grain in the region. Instead, we tentatively suggest that the Early Permian igneous activity in S Cambodia and SW Vietnam was linked to westwards-dipping subduction of the Paleo-Pacific Plate.

\section{Author statement and contributions}

Tod Waight: Conceptualization, writing - original draft, data manipulation. Michael B.W. Fyhn: Conceptualization, writing - original draft, funding acquisition, project administration. Tonny B. Thomsen: Formal analysis, data collection and manipulation, writing (review and editing). Tran Van Tri: Provided resources, background knowledge, and assisted with fieldwork. Lars H. Nielsen: Fieldwork, writing (review and editing), funding acquisition. Ioannis Abatzis: Fieldwork, writing (review and editing), funding acquisition. Dirk Frei: Formal analysis, data collection and manipulation, writing (review and editing).

\section{Declaration of Competing Interest}

The authors declare that they have no known competing financial interests or personal relationships that could have appeared to influence the work reported in this paper.

\section{Acknowledgements}

This study was supported by a number of research projects that were funded by Shell International Exploration \& Production, Geocenter
Denmark and DANIDA. The Vietnam Petroleum Institute (VPI) and Cambodian National Petroleum Agency (now General Department of Petroleum, Cambodia) helped organize fieldwork aided by the efficient and kind logistic help of Socheat Chea, Le C. Mai, Nguyen T. Huyen, Hoang A. Tuan, Ung Rany and Em Sokhom. Thanks to Jette Halskov, John Boserup and Mojagan Alaei, GEUS, who assisted with rock preparation, zircon separation and artwork. Tim Breitfeld and Takayuki Manaka are thanked for insightful and constructive reviews which improved and clarified our study.

\section{Supplementary material}

Supplementary data to this article can be found online at https://doi. $\operatorname{org} / 10.1016 /$ j.jseaes.2021.104902.

\section{References}

Anders, E., Grevesse, N., 1989. Abundances of the elements: Meteoric and solar. Geochim. Cosmochim. Acta 53, 197-214.

Atherton, M.P., Petford, N., 1993. Generation of sodium rich magmas from newly underplated basaltic crust. Nature 362, 144-146.

Barr, S.M., Charusiri, P., 2011. Volcanic rocks. In: Ridd, M.F., Barber, A.J., Crow, M.J. (Eds.), The Geology of Thailand. Geological Society, London, pp. 273-334.

Barr, S.M., MacDonald, A.S., Dunning, G.R., Ounchanum, P., Yaowanoiyothin, W., 2000. Petrochemistry, U-Pb (zircon) age, and palaeotectonic setting of the Lampang volcanic belt, northern Thailand. J. Geol. Soc. London 157, 553-563.

Barr, S.M., MacDonald, A.S., Ounchanum, P., Hamilton, M.A., 2006. Age, tectonic setting and regional implications of the Chiang Khong volcanic suite, northern Thailand. J. Geol. Soc. London 163, 1037-1046.

Booth, J., Sattayarak, N. 2011. Subsurface Carboniferous-Cretaceous geology of NE Thailand. In: M. F. Ridd, A. J. Barber, and M. J. Crow (Eds), The Geology of Thailand. Geol. Soc., London. pp. 185-222.

Breitfeld, H.T., Hall, R., Galin, T., Forster, M.A., BouDagher-Fadel, M.K., 2017. A Triassic to Cretaceous Sundaland-Pacific subduction margin in West Sarawak, Borneo. Tectonophysics 694, 35-56.

Breitfeld, H.T., Davies, L., Hall, R., Armstrong, R., Forster, M., Lister, G., Thirlwall, M., Grassineau, N., Hennig-Breitfeld, J., van Hattum, M.W.A., 2020. Mesozoic PaleoPacific subduction beneath SW Borneo: U-Pb geochronology of the Schwaner granitoids and the Pinoh Metamorphic Group. Front. Earth Sci. 8, 568715.

BRGM (Bureau de Recherches Géologiques et Minières), 1973. Carte Géologique de Reconnaissance de la République Khmère, Kampot, Scale 1:200,000. Service National des Mines, de la Géologie et du Pétrole, Republique Khmère.

Burton-Johnson, A., Macpherson, C.G., Millar, I.L., Whitehouse, M.J., Ottley, C.J., Nowell, G.M., 2020. A Triassic to Jurassic arc in north Borneo: Geochronology, geochemistry, and genesis of the Segama Valley Felsic Intrusions and the Sabah ophiolite. Gondwana Res. 84, 229-244.

Cawood, P.A., Leitch, E.C., Merle, R.E., Nemchin, A.A., 2011. Orogenesis without collision: Stabilizing the Terra Australis accretionary orogen, eastern Australia. GSA Bulletin 123, 2240-2255.

Cheng, R., Uchida, E., Katayose, M., Yarimizu, K., Shin, K.-C., Kong, S., Nakano, T., 2019. Petrogenesis and tectonic setting of Late Paleozoic to Late Mesozoic igneous rocks in Cambodia. J. Asian Earth Sci. 185, 104046.

Defant, M.J., Drummond, M.S., 1990. A model for trondhjemite-tonalite-dacite genesis and crustal growth via slab melting: Archean to modern comparisons. J. Geophys. Res. Solid Earth 95, 21503-21521.

Ewart, A., 1982. The mineralogy and petrology of Tertiary-Recent orogenic volcanic rocks: with special reference to the andesitic-basaltic compositional range. In Thorpe, R.S. (Ed) Andesites: Orogenic Andesites and Related Rocks, 25-98.

Faure, M., Nguyen, V.V., Hoai, L.T.T., Lepvrier, C., 2018. Early Paleozoic or Early-Middle Triassic collision between the South China and Indochina Blocks: The controversy resolved? Structural insights from the Kon Tum massif (Central Vietnam). J. Asian Earth Sci. 166, 162-180.

Ferrari, O.M., Hochard, C., Stampfli, G.M., 2008. An alternative plate tectonic model for the Palaeozoic-Early Mesozoic Palaeotethyan evolution of Southeast Asia (Northern Thailand-Burma). Tectonophysics 451, 346-365.

Frei, D., Gerdes, A., 2009. Precise and accurate in situ U-Pb dating of zircon with high sample throughput by automated LA-SF-ICPMS. Chem. Geol. 261, 261-270.

Frost, B.R., Barnes, C.G., Collins, W.J., Arculus, R.J., Ellis, D.J., Frost, C.D., 2001. A geochemical classification for granitic rocks. J. Petrol. 42, 2033-2048.

Fyhn, M.B.W., Boldreel, Lars O., Nielsen, Lars H., 2010a. Escape tectonism in the Gulf of Thailand: Paleogene left-lateral pull-apart rifting in the Vietnamese part of the Malay Basin. Tectonophysics 483 (3-4), 365-376.

Fyhn, M.B.W., Pedersen, S.A.S., Boldreel, L.O., Nielsen, L.H., Green, P.F., Dien, P.T., Huyen, L.T., Frei, D., 2010b. Palaeocene-early Eocene inversion of the PhuquocKampot Som Basin: SE Asian deformation associated with the suturing of Luconia. J. Geol. Soc. 167, 281-295.

Fyhn, M.B.W., Green, P.F., Bergman, S.C., Van Itterbeeck, J., Tri, T.V., Dien, P.T., Abatzis, I., Thomsen, T.B., Chea, S., Pedersen, S.A.S., Mai, L.C., Tuan, H.A., Nielsen, L.H. 2016., Cenozoic deformation and exhumation of the Kampot Fold Belt and implications for south Indochina tectonics. J. Geophys. Res.: Solid Earth, 121, doi: 10.1002/ 2016JB012847. 
Gao, S., Rudnick, R.L., Yuan, H.L., Liu, X.M., Liu, Y.S., Xu, W.L., Ling, W.L., Ayers, J., Wang, X.C., Wang, Q.H., 2004. Recycling lower continental crust in the North China craton. Nature 432, 892-897.

Gerdes, A., Zeh, A., 2006. Combined U-Pb and Hf isotope LA-(MC)-ICP-MS analyses of detrital zircons: comparison with SHRIMP and new constraints for the provenance and age of an Armorican metasediment in Central Germany. Earth Planet. Sci. Lett. 249, 47-61.

Ghani, A.A., Searle, M., Robb, L., Chung, S-L. 2013. Transitional I-S type characteristics in the Main Range Granite, Peninsular Malaysia. J. Asian Earth Sci. 76, 225-240.

Gillespie, M.R., Kendall, R.S., Leslie, A.G., Millar, I.L., Dodd, T.J.H., Kearsey, T.I., Bide, T. P., Goodenough, K.M., Dobbs, M.R., Lee, M.K.W., Chiam, L. 2019. The igneous rocks of Singapore: New insights to Palaeozoic and Mesozoic assembly of the Sukhothai Arc. J. Asian Earth Sci. 183, 103940.

Guo, F., Wu, Yangming, Zhang, B., Zhang, X., Zhao, L., Liao, J., 2021. Magmatic responses to Cretaceous subduction and tearing of the paleo-Pacific Plate in SE China: An overview. Earth Sci. Rev. 212 (2021), 103488.

Hall, R., 1996. Reconstructing Cenozoic SE Asia. In Hall, R. \& Blundell, D. (eds), Tectonic Evolution of Southeast Asia, Geological Society Special Publication No. 106, pp. 153184.

Hall, R., 2012. Late Jurassic-Cenozoic reconstructions of the Indonesian region and the Indian Ocean. Tectonophysics 570-571, 1-41.

Hara, H., Tokiwa, T., Kurihara, T., Charoentitirat, T., Ngamnithiporn, A., Visetnat, K., Tominaga, K., Kamata, Y., Ueno, K., 2018. Permian-Triassic back-arc basin development in response to Paleo-Tethys subduction, Sa Kaeo-Chanthaburi area in Southeastern Thailand. Gondwana Res. 64, 50-66.

Hara, H., Ito, T., Tokiwa, T., Kong, S., Lim, P., 2020. The origin of the Pailin Crystalline Complex in western Cambodia, and back-arc basin development in the Paleo-Tethys Ocean. Gondwana Res. 82, 299-316.

Hellstrom, J., Paton, C., Woodhead, J., Hergt, J., 2008. Iolite: software for spatially resolved LA-(quad and MC) ICPMS analysis. Mineral. Assoc. Canada short course series 40, 343-348.

Hennig, J., Breitfeld, H.T., Hall, R., Nugraha, A.M.S., 2017. The Mesozoic tectonomagmatic evolution at the Paleo-Pacific subduction zone in West Borneo. Gondwana Res. 48, 292-310.

Hoa, N. N. (Ed.) 1996. Geology and Mineral Resources Map of Vietnam, Map sheet: Phu Quoc-Ha Tien, (C-48-XIV \& C-48-XV). 1:200,000, with explanatory note (in Vietnamese and English), Dep. Geol. and Miner. of Viet Nam, Hanoi, Vietnam.

Henning-Breitfeld, J., Breitfeld, H.T., Sang, D.Q., Vinh, M.K., Long, T.V., Thirlwall, M., Cuong, T.X., 2021. Ages and character of igneous rocks of the Da Lat Zone in SE Vietnam and adjacent offshore regions (Cuu Long and Nam Con Son basins). J. Asian Earth Sci. 218 (104878).

Hu, L., Cawood, P.A., Du, Y., Yang, J., Jiao, L., 2015. Late Paleozoic to Early Mesozoic provenance record of Paleo-Pacific subduction beneath South China. Tectonics 34, 986-1008.

Jackson, S.E., Pearson, N.J., Griffin, W.L., Belousova, E.A., 2004. The application of laser ablation-inductively coupled plasma mass spectrometry to in situ U-Pb zircon geochronology. Chem. Geol. 211, 47-69.

Jiang, H., Li, W-Q., Z, K-D., Zhang, D., Jiang, S-Y., 2021. Middle Triassic diorites from the Loei Fold Belt, NE Thailand: Petrogenesis and tectonic implications in the context of Paleotethyan subduction. Lithos 382-383, 105955

Lan, C.-Y., Chung, S.-L., Long, T.V., Lo, C.-H., Lee, T.-Y., Mertzman, S.A., Shen, J.J.-S., 2003. Geochemical and $\mathrm{Sr}-\mathrm{Nd}$ isotopic constraints from the Kontum massif, centra Vietnam on the crustal evolution of the Indochina block. Precambr. Res. 122, 7-27.

Le Bas, M.J., Le Maitre, R.W., Streckeisen, A., Zanettin, B., 1986. A chemical classification of volcanic rocks based on the total alkali-silica diagram. J. Petrol. 27, 745-750.

Li, Z.-X., Li, X.-H., 2007. Formation of the 1300-km-wide intracontinental orogen and postorogenic magmatic province in Mesozoic South China: A flat-slab subduction model. Geology 35 (2), 179. https://doi.org/10.1130/G23193A.110.1130/2007041.

Li, X.-H., Li, Z.-X., Li, W.-X., Wang, Y., 2006. Initiation of the Indosinian Orogeny in South China: Evidence for a Permian magmatic arc on Hainan Island. J. Geol. 114, 341-353.

Ludwig, K.R., 2003. Isoplot/Ex 3.00. A geochronological toolkit for Microsoft Excel. Special Publication, vol. 4. Berkeley Geochronological Center, Berkeley, CA.

Metcalfe, I., 1996. Pre-Cretaceous evolution of SE Asian terranes. In: Hall, R., Blundell, D. (Eds.), Tectonic Evolution of Southeast Asia, Geological Society Special Publication No. 106, 97-122.

Metcalfe, I., 2009. Comment on "An alternative plate tectonic model for the PalaeozoicEarly Mesozoic Palaeotethyan evolution of Southeast Asia (Northern Thailand-Burma)" by O.M. Ferrari, C. Hochard \& G.M. Stampfli, Tectonophysics 451, 346-365.

Metcalfe, I., 2011. Tectonic framework and Phanerozoic evolution of Sundaland. Gondwana Res. 19 (1), 3-21.

Metcalfe, I., 2013a. Tectonic evolution of the Malay Peninsula. J. Asian Earth Sci. 76, 195-213.

Metcalfe, I., 2013b. Gondwana dispersion and Asian accretion: Tectonic and palaeogeographic evolution of eastern Tethys. J. Asian Earth Sci. 66, 1-33.

Metcalfe, I., 2017. Tectonic evolution of Sundaland. Bull. Geol. Soc. Malaysia 63, 27-60.

Metcalfe, I., 2021. Multiple Tethyan ocean basins and orogenic belts in Asia. Gondwana Res. https://doi.org/10.1016/j.gr.2021.01.012.

Middlemost, E.A.K., 1994. Naming materials in the magma/igneous rock system. Earth Sci. Rev. 37, 215-224.
Morley, C.K., 2012. Late Cretaceous-Early Palaeogene tectonic development of SE Asia. Earth Sci. Rev. 115 (1-2), 37-75.

Nesbitt, H.W., Young, G.M., 1982. Early Proterozoic climates and plate motions inferred from major element chemistry of lutites. Nature 299 (5885), 715-717.

Ng, SW.-P., Whitehouse, M.J., Searle, M.P., Robb, L.J., Ghani, A.A., Chung, S.-L., Oliver, G.J.H., Sone, M., Gardiner, N.J., Roselee, M.H., 2015. Petrogenesis of Malaysian granitoids in the Southeast Asian tin belt: Part 2. U-Pb zircon geochronology and tectonic model. Geol. Soc. Am. Bull. 127, 1238-1258.

Nguyen, A.T., Nguyen, T.C., 2017. The Triassic suture between Indochina and Sibumasu block -the first discovery of a backarc basin within Vietnam territory. Sci. Technol. Develop. J. -Sci. Earth Environ. 1, 14-22 (in Vietnamese with English abstract).

Nguyen, T.T.B., Satir, M., Siebel, W., Chen, F., 2004. Granitoids in the Dalat zone, southern Vietnam: age constraints on magmatism and regional geological implications. Int. J. Earth Sci. 93, 329-340.

Nong, A.T.Q., Hauzenberger, C.A., Gallhofer, D., Dinh, S.Q., 2021. Geochemistry and zircon U-Pb geochronology of Late Mesozoic igneous rocks from SW Vietnam - SE Cambodia: Implications for episodic magmatism in the context of the Paleo-Pacific subduction. Lithos 390-391, 106101. https://doi.org/10.1016/j.lithos.2021.106101.

Nualkhao, P., Takahashi, R., Imai, A., Charusiri, P., 2018. Petrochemistry of granitoids along the Loei Fold Belt, Northeastern Thailand. Resour. Geol. 68, 395-424.

Oliver, G., Zaw, K., Hotson, M., Meffre, S., Manka, T., 2014. U-Pb zircon geochronology of Early Permian to Late Triassic rocks from Singapore and Johor: A plate tectonic reinterpretation. Gondwana Res. 26 (1), 132-143.

Paton, C., Hellstrom, J., Paul, B., Woodhead, J., Hergt, J., 2011. Iolite: Freeware for the visualisation and processing of mass spectrometric data. J. Anal. At. Spectrom. 26, 2508-2518.

Pearce, J.A., 1982. Trace element characteristics of lavas from destructive plate boundaries. In: Thorpe, R.S. (Ed.), Andesites. JohnWiley, Chichester, pp. 525-547.

Pearce, J.A., Harris, N.B.W., Tindle, A.G., 1984. Trace element discrimination diagrams for the tectonic interpretation of granitic rocks. J. Petrol. 25 (4), 956-983.

Petrus, J.A., Kamber, B.S., 2012. VizualAge: A novel approach to laser ablation ICP-MS U-Pb geochronology data reduction. Geostand. Geoanal. Res. 36, 247-270.

Qian, X., Feng, Q., Wang, Y., Yang, W., Chonglakmani, C., Monjai, D., 2016. Petrochemistry and tectonic setting of the middle Triassic arc-like volcanic rocks in the Sayabouli Area, NW Laos. J. Earth Sci. 27, 365-377.

Qian, X., Feng, Q., Wang, Y., Zhao, T., Zi, J.-W., Udchachon, M., Wang, Y., 2017. Late Triassic post-collisional granites related to Paleotethyan evolution in SE Thailand: Geochronological and geochemical constraints. Lithos 286-287, 440-453.

Roger, F., Leloup, P.H., Jolivet, M., Lacassin, R., Trinh, P.T., Brunel, M., Seward, D., 2000. Long and complex thermal history of the Song Chay metamorphic dome (Northern Vietnam) by multi-system geochronology. Tectonophysics 321 (4), 449-466.

Roger, F., Maluski, H., Leyreloup, A., Lepvrier, C., Truong Thi, P., 2007. U-Pb dating of high temperature episodes in the Kon Tum Massif (Vietnam). J. Asian Earth Sci. 30, 565-572.

Rossignol, C., Bourquin, S., Poujol, M., Hallot, E., Dabard, M.-P., Nalpas, T., 2016. The volcaniclastic series from the Luang Prabang Basin, Laos: A witness of a Triassic magmatic arc? J. Asian Earth Sci. 120, 159-183.

Saesaengseerung, D., Agematsu, S., Sashida, K., Sardsud, A., 2009. Discovery of Lower Permian radiolarian and conodont faunas from the bedded chert of the Chanthaburi area along the Sra Kaeo suture zone, eastern Thailand. Paleontolog. Res. 13 (2), $119-138$.

Searle, M.P., Whitehouse, M.J., Robb, L.J., Ghani, A.A., Hutchison, C.S., Sone, M., Ng, S. W.-P., Roselee, M.H., Chung, S.-L., Oliver, G.J.H., 2012. Tectonic evolution of the Sibumasu-Indochina terrane collision zone in Thailand and Malaysia: constraints from new U-Pb zircon chronology of SE Asian tin granitoids. J. Geol. Soc. 169 (4), 489-500.

Sevastjanova, I., Clements, B., Hall, R., Belousova, E.A., Griffin, W.L., Pearson, N., 2011. Granitic magmatism, basement ages, and provenance indicators in the Malay Peninsula: insights from detrital zircon U-Pb and Hf-isotope data. Gondwana Res. 19 (4), 1024-1039.

Sewell, R.J., Campbell, S.D.G., 1997. Geochemistry of coeval Mesozoic plutonic and volcanic suites in Hong Kong. J. Geol. Soc., London 154, 1053-1066.

Shellnutt, J.G., Lan, C.-Y., Van Long, T., Usuki, T., Yang, H.-J., Mertzman, S.A., Iizuka, Y., Chung, S.-L., Wang, K.-L., Hsu, W.-Y., 2013. Formation of Cretaceous Cordilleran and post-orogenic granites and their microgranular enclaves from the Dalat zone, southern Vietnam: Tectonic implications for the evolution of Southeast Asia. Lithos 182-183, 229-241.

Slama, J., Kosler, J., Condon, D.J., Crowley, J.L., Gerdes, A., Hanchar, J.M., Horstwood, M.S.A., Morris, G.A., Nasdala, L., Norberg, N., Schaltegger, U., Schoene, N., Tubrett, M.N., Whitehouse, M.J., 2008. Plešovice zircon - a new natural reference material for U-Pb and Hf isotopic microanalysis. Chem. Geol. 249, 1-35.

Sone, M., Metcalfe, I., 2008. Parallel Tethyan Sutures in mainland SE Asia: new insights for Palaeo-Tethys closure. Compte Rendus Geosci. 340, 166-179.

Sone, M., Metcalfe, I., Chaodumrong, P., 2012. The Chanthaburi terrane of southeastern Thailand: Stratigraphic confirmation as a disrupted segment of the Sukhothai Arc. J. Asian Earth Sci. 61, 16-32.

Spandler, C., Worden, K., Arculus, R., Eggins, S., 2005. Igneous rocks of the Brook Street Terrane, New Zealand: Implications for Permian tectonics of eastern Gondwana and magma genesis in modern intra-oceanic volcanic arcs. New Zealand J. Geol. Geophys. 48, 167-183. 
Thanh, T.V., Hieu, P.T., Minh, P., Nhuan, D.V., Thuy, N.T.B., 2019. Late Permian-Triassic granitic rocks of Vietnam: the Muong Lat example. Int. Geol. Rev. 61, 1823-1841.

Thuy, N.T.B., Satir, M., Siebel, W., Vennemann, T., Long, T.V., 2004. Geochemical and isotopic constraints on the petrogenesis of granitoids from the Dalat zone, southern Vietnam. J. Asian Earth Sci. 23, 467-482.

Tran, V.T., Vu, K. (Eds.), 2011. Geology and Earth Resources of Vietnam. Publishing House for Science and Technology, Hanoi. $634 \mathrm{pp}$.

Tran, T., Faure, M., Nguyen, V.V., Bui, H.H., Fyhn, M.B.W., Nguyen, T.Q., Lepvrier, C., Thomsen, T.B., Tani, K., Charusiri, P., 2020. Neoproterozoic to Early Triassic tectono-stratigraphic evolution of Indochina and adjacent areas: A review with new data. J. Asian Earth Sci. 191, 104231.

Wang, Y., Qian, X., Cawood, P.A., Liu, H., Feng, Q., Zhao, G., Zhang, Y., He, H., Zhang, P., 2018. Closure of the East Paleotethyan Ocean and amalgamation of the Eastern Cimmerian and Southeast Asia continental fragments. Earth Sci. Rev. 186, 195-230.

Whalen, J.B., Currie, K.L., Chappell, B.W., 1987. A-type granites: geochemical characteristics, discrimination and petrogenesis. Contrib. Miner. Petrol. 95, 407-419.

Wiedenbeck, M., Allé, P., Corfu, F., Griffin, W.L., Meier, M., Oberli, F., von Quadt, A., Roddick, J.C., Spiegel, W., 1995. Three natural zircon standards for U-Th-Pb, Lu-Hf, trace element and REE analyses. Geostandards Newslett. 19, 1-23.

Wiedenbeck, M., Hanchar, J.M., Peck, W.H., Sylvester, P., Valley, J., Whitehouse, M., Kronz, A., Morishita, Y., Nasdala, L., Fiebig, J., Franchi, I., Girard, J.-P., Greenwood, R.C., Hinton, R., Kita, N., Mason, P.R.D., Norman, M., Ogasawara, M., Piccoli., P.M., Rhede, D., Satoh, H., Schulz-Dobrick, B., Skår, Ø., Spicuzza, M.J., Terada, K., Tindle,
A., Togashi, S., Vennemann, T., Xie, Q. and Zheng, Y.-F. 2004. Further characterisation of the 91500 zircon crystal. Geostandards Geoanal. Res. 28, 9-39.

Xu, C., Shi, H., Barnes, C.G., Zhou, Z., 2016. Tracing a late Mesozoic magmatic arc along the Southeast Asian margin from the granitoids drilled from the northern South China Sea. Int. Geol. Rev. 58, 71-94.

Xu, C., Zhang, L., Shi, H., Brix, M.R., Huhma, H., Chen, L., Zhang, M., Zhou, Z., 2017. Tracing an Early Jurassic magmatic arc from South to East China Seas. Tectonics 36, 466-492.

Yang, W., Qian, X., Feng, Q., Shen, S., Chonglakmani, C., 2016. Zircon U-Pb Geochronological Evidence for the Evolution of the Nan-Uttaradit Suture in Northern Thailand. J. Earth Sci. 27, 378-390.

Zahirovic, S., Matthews, K.J., Flament, N., Müller, D., Hill, K.C., Seton, M., Gurnis, M., 2016. Tectonic evolution and deep mantle structure of the eastern Tethys since the latest Jurassic. Earth Sci. Rev. 162, 293-337.

Zaw, K., Meffre, S., Lai, C.-K., Burrett, C., Santosh, M., Graham, I., Manaka, T., Salama, A., Kamvong, T., Cromie, P., 2014. Tectonics and metallogeny of mainland Southeast Asia - A review and contribution. Gondwana Res. 26 (2014), 5-30.

Zaw, K., Swi, W., Barber, A.J., Crow, M.J., New, Y.Y., 2017. Introduction to the geology of Myanmar. In: Barber, A.J., Zaw, K., Crow, M.J., (Eds) Myanmar Geology, Resources and Tectonics, Geol. Soc. London, Memoir 48, 1-18.

Zhou, X.M., Li, W.X., 2000. Origin of Late Mesozoic igneous rocks in Southeastern China: implications for lithosphere subduction and underplating of mafic magmas. Tectonophysics 326 (3-4), 269-287.

Zhou, X., Sun, T., Shen, W., Shu, L., Niu, Y., 2006. Petrogenesis of Mesozoic granitoids and volcanic rocks in South China: A response to tectonic evolution. Episodes 29 (1), 26-33. 